\title{
SUBMANIFOLDS IN HYPER-KÄHLER GEOMETRY
}

\author{
ROBERT BRYANT AND REESE HARVEY
}

\section{INTRODUCTION}

A calibration $\phi$ is a differential form on a Riemannian manifold with two additional properties. First, the form should be closed under exterior differentiation. Second, it should be less than or equal to the volume form on each oriented submanifold (of the same dimension as the degree of the form $\phi$ ). Each calibration $\phi$ determines a geometry of submanifolds, namely those oriented submanifolds for which $\phi$ restricts to be exactly the volume form. Such submanifolds are called $\phi$-submanifolds. The Fundamental Lemma of the theory of calibrations says that each $\phi$-submanifold is homologically area minimizing.

A Kähler form provides the most important classical example of a calibration. In this case the $\phi$-submanifolds are just the complex submanifolds of dimension one. One of the most interesting nonclassical examples of a calibration, introduced in Harvey and Lawson [HL], is a 4 -form $\Phi$ on euclidean $\mathbf{R}^{8}$ called the Cayley 4-form. This 4-form $\Phi$ has an elegant description in terms of the algebra of octonians $\mathbf{O}$, and is fixed by the subgroup $\operatorname{Spin}(7)$ of the group of all orthogonal transformations on $\mathbf{O}$. As such, it would appear unlikely that $\Phi$ would have higher dimensional generalizations. The purpose of this paper is to provide the higher dimensional analogue.

The Cayley form $\Phi$ on $\mathbf{R}^{8} \equiv \mathbf{O}$ can also be considered in a very natural way as a 4-form on $\mathbf{H}^{2}$, the quaternionic plane. After choosing to distinguish the scalar quaternion $K$, the 4 -form can be expressed as

$$
\Phi_{K} \equiv-\frac{1}{2} \omega_{I}^{2}-\frac{1}{2} \omega_{J}^{2}+\frac{1}{2} \omega_{K}^{2}
$$

where $\omega_{I}, \omega_{J}$, and $\omega_{K}$ are the Kähler forms associated with the complex structures, $R_{I}, R_{J}$, and $R_{K}$ respectively, obtained by right quaternionic scalar multiplication.

Now (1.1) provides a 4-form $\Phi_{K}$ on $\mathbf{H}^{n}$ for all $n$. When $n \geq 3$ the stabilizer of $\Phi_{K}$ in $O(4 n)$ is the subgroup $\mathrm{Sp}(n) \cdot\left(S^{1} \cup R_{I} S^{1}\right)$ of the quaternionic unitary group $\mathrm{Sp}(n) \cdot \mathrm{Sp}(1)$ (see Lemma 2.14). The natural geometric setting for $\Phi_{K}$ is obtained by replacing $\mathbf{H}^{n}$ by any hyper-Kähler manifold (see $\S 8$ ).

Received by the editors October 21, 1987.

1980 Mathematics Subject Classification (1985 Revision). Primary 53C40, 53C42, 53C55.

This work was partially funded by National Science Foundation Grants PHY-8705565 and DMS 8601853. 
The main result (Theorem 2.27) of this paper has two parts. First, $\Phi_{K}$ is a calibration. Second,

$$
G\left(\Phi_{K}\right) \equiv\left\{\xi \in G_{R}\left(4, \mathbf{H}^{n}\right): \Phi_{K}(\xi)=1\right\},
$$

the set of possible tangent 4-planes for which $\Phi_{K}$ is exactly the volume form, can be put in a "normal form" with respect to the automorphism group $\operatorname{Sp}(n)$. $\left(S^{\prime} \cup R_{I} S^{\prime}\right.$ ) of $G\left(\Phi_{K}\right)$ (see (2.31)). In particular, if $\Phi_{K}(\xi)=1$ then the real span of $\xi$ is contained in some quaternionic 2-plane. This normal form, even in the [HL] case $n=2$ is new, and provides additional insight into the geometry of $\Phi$-submanifolds of $\mathbf{O}$. We mention one example. Recall from [HL] that a submanifold $M$ of $\mathbf{O}$ with the property that it is $I_{\theta} \equiv \cos \theta R_{i}+\sin \theta R_{j}$ anticomplex at each point is a $\Phi$-submanifold of $\mathbf{O}$; even if the complex structure $I_{\theta}$ varies. However, Proposition 7.1 states that $\theta$ must be constant on each connected component of $M$.

The fundamental 4-form $\Phi_{K}$ on $\mathbf{H}^{n}$ is also of interest from the following point of view. Given a calibration, $\phi$, the larger the set $G(\phi)$ of possible $p$ planes $\xi$ with $\phi(\xi)=1$, the richer the geometry of $\phi$-submanifolds. Thus calibrations $\phi \in \Lambda^{p}\left(\mathbf{R}^{n}\right)^{*}$ with $G(\phi)$ maximal are of particular interest. The known (nontrivial) examples of such maximal calibrations are:

(1) The standard Kähler form $\omega$ and $(1 /(n-1) !) \omega^{n-1}$ on $\mathbf{C}^{n}$,

(2) $\operatorname{Re}\left(d z_{1} \wedge d z_{2} \wedge d z_{3}\right)$; the special Lagrangian calibration on $\mathbf{C}^{3}$,

(3) $\phi$ and $\psi$; the associative and coassociative calibrations on $\operatorname{Im} \mathbf{O}$,

(4) $\Phi$, the Cayley calibration on $\mathbf{O}$.

Note all but the first calibration is only for a specific dimension. In $\S 4$, the 4-form $\Phi_{K}$ is proved to be maximal. In addition, $\Phi_{K}$ provides a natural extension of the associative 3-form $\phi$ from $\operatorname{Im} \mathbf{O} \equiv \operatorname{Im} \mathbf{H} \oplus \mathbf{H}$ to a generalized associative 3-form $\phi_{K}$ on $\operatorname{Im} \mathbf{H} \oplus \mathbf{H}^{n}$ for all $n$, which is also shown to be maximal in $\S 4$.

\section{The Fundamental 4-FORM ON $\mathbf{H}^{n}$}

We shall consider $\mathbf{H}^{n}$, the set of columns of height $n$ of quaternions, as a (right) quaternion vector space. We identify the space of $n \times n$ matrices over $\mathbf{H}$, denoted $M_{n}(\mathbf{H})$, with the space of $\mathbf{H}$-linear maps of $\mathbf{H}^{n}$, denoted End $_{H}\left(\mathbf{H}^{n}\right)$, by letting $A \in M_{n}(\mathbf{H})$ act on the left of $p \equiv{ }^{t}\left(p_{1}, \ldots, p_{n}\right) \in \mathbf{H}^{n}$. Let $\varepsilon(p, q$ ) (or ' $\bar{p} q$ ) denote the standard quaternionic hermitian bilinear form on $\mathbf{H}^{n}$,

$$
\varepsilon(p, q) \equiv \sum_{l=1}^{n} \bar{p}^{l} q^{l} .
$$

The special quaternionic unitary group

$$
\operatorname{Sp}(n) \equiv\left\{A \in M_{n}(\mathbf{H}): \varepsilon(A p, A q)=\varepsilon(p, q)\right\}
$$

is also sometimes called the symplectic group. $\operatorname{Re} \varepsilon(p, q)(\equiv\langle p, q\rangle)$ equals the standard euclidean inner product on $\mathbf{R}^{4 n} \cong \mathbf{H}^{n}$. For each unit imaginary 
quaternion $u \in S^{2} \subset \operatorname{Im} \mathbf{H}$, right multiplication by $u, R_{u} p \equiv p u$, defines an orthogonal complex structure on $\mathbf{R}^{4 n}$. Let

$$
\omega_{u}(p, q) \equiv\langle u, \varepsilon(p, q)\rangle=\left\langle R_{u} p, q\right\rangle
$$

denote the standard Kähler form with respect to the complex structure $R_{u}$. Note that each of the forms $\omega_{u}$ is fixed by $\operatorname{Sp}(n)$. Let $I \equiv R_{i}, J \equiv R_{j}$, and $K \equiv R_{k}$ denote the standard basis for the pure imaginary scalars, $\operatorname{Im} \mathbf{H}$, acting on $\mathbf{H}^{n}$. Then

$$
\varepsilon(p, q)=\langle p, q\rangle+i \omega_{I}(p, q)+j \omega_{J}(p, q)+k \omega_{K}(p, q) .
$$

The group $\operatorname{Sp}(1) \equiv S^{3} \equiv\{v \in \mathbf{H}:\|v\|=1\}$ of unit scalars acts on $\mathbf{H}^{n}$ on the right. The induced action on $\operatorname{Im} \mathbf{H} \cong\left\{\omega_{u}: u \in \operatorname{Im} \mathbf{H}\right\}$ is given by:

$$
R_{v}^{*} \omega_{u}=\omega_{v u \bar{v}}, \quad \text { or } u \rightarrow v u \bar{v} \equiv \chi(v) u
$$

where

$$
\chi: \mathrm{Sp}(1) \rightarrow \mathrm{SO}(3) \equiv \mathrm{SO}(\operatorname{Im} \mathbf{H})
$$

is the standard double cover of $\mathrm{SO}(3)$ by $\mathrm{Sp}(1) \cong \operatorname{Spin}(3)$. To prove (2.4) note that

$$
\left(R_{v}^{*} \omega_{u}\right)(p, q)=\omega_{u}(p v, q v)=\langle p v u, q v\rangle=\langle p v u \bar{v}, q\rangle=\omega_{v u \bar{v}}(p, q) .
$$

Remark 2.5. Identify $A \equiv\left(a_{i j}\right) \in \operatorname{Sym}_{3}(\mathbf{R}) \subset M_{3}(\mathbf{R})$ with

$$
\begin{aligned}
\widetilde{A} \equiv & a_{11} \omega_{I}^{2}+a_{12} \omega_{I} \wedge \omega_{J}+a_{13} \omega_{I} \wedge \omega_{K} \\
& +a_{21} \omega_{J} \wedge \omega_{I}+a_{22} \omega_{J}^{2}+a_{23} \omega_{J} \wedge \omega_{K} \\
& +a_{31} \omega_{K} \wedge \omega_{I}+a_{32} \omega_{K} \wedge \omega_{J}+a_{33} \omega_{K}^{2} .
\end{aligned}
$$

Then it follows easily from (2.4) that the action of $\operatorname{Sp}(1)$ on $\operatorname{Sym}_{3}(\mathbf{R})$ is just the usual action of $\mathrm{SO}(3)$ on $\operatorname{Sym}_{3}(\mathbf{R})$ sending $A \rightarrow g A g^{t}$, with $g=\chi(v) \in$ $\mathrm{SO}(3)$.

Note that the group $\operatorname{Sp}(1) \equiv S^{3} \subset \mathbf{H}$, of unit scalars, does not belong to $\operatorname{Sp}(n)$. In fact, the intersection is $Z_{2} \equiv\{ \pm I d\}$. The enhancement of $\operatorname{Sp}(n)$ by the unit scalars, i.e., the subgroup of $\mathrm{GL}_{\mathbf{R}}\left(\mathbf{H}^{n}\right)$ generated by $\operatorname{Sp}(n) \times \operatorname{Sp}(1)$ is

$$
\mathrm{Sp}(n) \cdot \mathrm{Sp}(1) \equiv \mathrm{Sp}(n) \times \mathrm{Sp}(1) / Z_{2},
$$

which we will refer to as the quaternionic unitary group. By Remark 2.5, the 4-form

$$
\omega_{I}^{2}+\omega_{J}^{2}+\omega_{K}^{2}
$$

is fixed by $\mathrm{Sp}(n) \cdot \mathrm{Sp}(1)$. In fact, if $A \in \mathrm{GL}_{\mathbf{R}}\left(\mathbf{H}^{n}\right)$ fixes $\frac{1}{2} \omega_{I}^{2}+\frac{1}{2} \omega_{J}^{2}+\frac{1}{2} \omega_{K}^{2}$ then $A \in \mathrm{Sp}(n) \cdot \mathrm{Sp}(1)$ (see Bryant and Harvey [BH]) so that this 4-form determines the quaternionic unitary group $\mathrm{Sp}(n) \cdot \mathrm{Sp}(1)$. This is not the 4-form of primary interest in this paper (although we shall compute its comass in §6).

In order to define the 4-form of primary interest in this paper we must first choose one of the complex structures $R_{u}$ (with $u \in S^{2} \subset \operatorname{Im} H$ ) on $H^{n}$. It is convenient to distinguish $K \equiv R_{K}$. 
Definition 2.9. The fundamental 4-form $\Phi_{K}$ determined by the choice $K$ is

$$
\Phi_{K} \equiv-\frac{1}{2} \omega_{I}^{2}-\frac{1}{2} \omega_{J}^{2}+\frac{1}{2} \omega_{K}^{2} \in \Lambda^{4}\left(R^{4 n}\right)^{*} \equiv \Lambda^{4}\left(\mathbf{H}^{n}\right)^{*}
$$

Remark. Actually, we have available a 2-sphere of fundamental 4-forms on $\mathbf{R}^{4 n} \cong \mathbf{H}^{n}$, denoted $\Phi_{u}$ with $u \in S^{2} \subset \operatorname{Im} \mathbf{H}$.

Since the action of $\operatorname{Sp}(1)$ (via right scalar multiplication) on $\left\{\omega_{u}: u \in \operatorname{Im~} \mathbf{H}\right\}$ is given by (2.4), the two circles:

$$
\begin{gathered}
S^{1} \equiv\left\{(\cos t)+(\sin t) R_{K}: t \in R\right\} \\
R_{I} S^{1} \equiv\left\{(\cos t) R_{I}+(\sin t) R_{J}: t \in R\right\}
\end{gathered}
$$

fix the fundamental 4-form $\Phi_{K}$. Moreover, since the action of $\mathrm{Sp}(1)$ on a form $\widetilde{A}$ defined by (2.6), with $A \in \operatorname{Sym}_{3}(R)$, is the same as the action of $\mathrm{SO}(3)$ on $\operatorname{Sym}_{3}(\mathbf{R})$, the subgroup of $\operatorname{Sp}(1)$ that fixes $\Phi_{K}$ is exactly:

$$
S^{1} \cup R_{I} S^{1}
$$

Thus the group

$$
\mathrm{Sp}(n) \cdot\left(S^{1} \cup R_{I} S^{1}\right), \quad \text { with } S^{1} \equiv\left\{e^{K t}: t \in \mathbf{R}\right\},
$$

fixes the fundamental 4-form $\Phi_{K}$.

Lemma 2.14. Let $H \equiv\left\{g \in \operatorname{SO}\left(\mathbf{H}^{n}\right): g^{*} \Phi_{K}=\Phi_{K}\right\}$ denote the subgroup of orthogonal transformations on $\mathbf{H}^{n}$ which fix $\Phi_{K}$.

$$
\text { If } n=2 \text { then } H=\operatorname{Spin}(7) .
$$

Proof $(n>2)$. The identity component $H^{0}$ of $H$ contains $\operatorname{Sp}(n)$ and hence acts transitively on $S^{4 n-1} \subseteq H^{n}$. By a well-known theorem of Borel, it follows that $H^{0}$ must be one of $\mathrm{Sp}(n), \mathrm{Sp}(n) \cdot P$ (where $P$ is a subgroup of $\mathrm{Sp}(1)$ ), $\mathrm{SU}(2 n), U(2 n), \mathrm{SO}(4 n)$, or $\operatorname{Spin}(9)$ (if $n=4$ ). The groups $\mathrm{SO}(4 n)$ and $\operatorname{Spin}(9)$ are ruled out since they do not fix any 4 -form on $\mathbf{R}^{4 n}$. Note that the assumption $n>2$ automatically rules out the possibility $H=\operatorname{Spin}(7)$. Similarly it is easy to show that $\operatorname{SU}(2 n)$ does not fix $\Phi_{K}$. Thus, the only remaining possibility is $H^{0}=\operatorname{Sp}(n) \cdot P$ where $P \subseteq \mathrm{Sp}(1)$ is a (connected) subgroup. Since $H^{0}$ contains the circle $S^{1} \equiv\left\{e^{K t}: t \in \mathbf{R}\right\}$ and does not contain $\{e\} \cdot \mathrm{Sp}(1)$, it follows that $H^{0}=\mathrm{Sp}(n) \cdot S^{1}$. Now $H^{0}$ is a normal subgroup of $H$. It easily follows that $\operatorname{Sp}(n)$ is a normal subgroup of $H$. However, since $\operatorname{Sp}(n)$ has no outer automorphisms, it is not difficult to show that any element of $H$ can be written in the form $h=A z$ where $A \in \operatorname{Sp}(n)$ and $z$ commutes with $\mathrm{Sp}(n)$. It follows that $z \in \mathrm{Sp}(1)$. Thus $H \subseteq \mathrm{Sp}(n) \cdot \operatorname{Sp}(1)$. Since $R_{I} \in H$, we see that $H$ properly contains $H^{0}$. In fact, we must have $H=\operatorname{Sp}(n) \cdot\left(S^{1} \cup R_{I} S^{1}\right)$. 
When $n=2$ the form $\Phi_{K}$ is well understood in terms of the octonians. The algebra of octonians $\mathbf{O}$ equals $\mathbf{H} \oplus \mathbf{H} e \equiv\{a+b e: a, b \in \mathbf{H}\}$, with the octonian multiplication defined by

$$
(a+b e)(c+d e)=a c-\bar{d} b+(b \bar{c}+d a) e .
$$

The Cayley form $\Phi \in \Lambda^{4} \mathbf{O}$ is defined by

$$
\Phi(x, y, z, w) \equiv\left\langle x, \frac{1}{2}[y(\bar{z} w)-w(\bar{z} y)]\right\rangle .
$$

This form is a calibration (see Harvey and Lawson [HL]), i.e.,

$$
\Phi(x \wedge y \wedge z \wedge w) \leq|x \wedge y \wedge z \wedge w|
$$

Let CAY denote $\left\{\xi \in G_{\mathbf{R}}(4, \mathbf{O}): \Phi(\xi)=|\xi|\right\}$, the set of oriented real 4-planes in $O$ where equality occurs in (2.19). The automorphism group $\operatorname{Spin}(7)$ acts transitively on CAY (see [HL]). Also, several descriptions of CAY are given in [HL].

If we let $I \equiv R_{i}(\mathbf{O})$ (right octonian multiplication by $i$ ) and $J \equiv R_{j}(\mathbf{O})$ (right octonian multiplication by $j$ ) then this imposes a quaternionic structure on $\mathbf{O} \cong \mathbf{H}^{2}$. However, note that $(a+b e) i \neq a i+(b i) e$ and that $((a+b e) i) j \neq$ $(a+b e) k$. Thus the quaternionic structure $\mathbf{O} \cong \mathbf{H}^{2}$ is not given by the isomorphism $\mathbf{O} \equiv \mathbf{H} \oplus \mathbf{H} e$. Instead, identify $(a, b) \in \mathbf{H}^{2}$ with

$$
L(a, b) \equiv a+(k b \bar{k}) e \in \mathbf{O} .
$$

Since

$$
(a+(k b \bar{k}) e) c=a c+(k b \bar{k} \bar{c}) e
$$

we see that under this identification, $L$, the quaternionic structures on $\mathbf{H}^{2}$ and $\mathbf{O}$ agree. That is,

$$
R_{i}(\mathbf{O}) L(a, b)=L R_{i}(a, b), \quad R_{j}(\mathbf{O}) L(a, b)=L R_{j}(a, b)
$$

so that $L: \mathbf{H}^{2} \rightarrow \mathbf{O}$ is a quaternionic linear isomorphism.

Lemma 2.21. Under the quaternionic linear isomorphism $L: \mathbf{H}^{2} \rightarrow \mathbf{O}$, defined by (2.20), the Cayley calibration $\Phi \in \Lambda^{4} \mathbf{O}$ pulls back to $\Phi_{K} \in \Lambda^{4} \mathbf{H}^{2}$.

Proof. Let $x \equiv(a, b) \in \mathbf{H}^{2}$, and let $\omega^{1}, \ldots, \omega^{8}$ denote the standard basis for $\Lambda^{1} \mathbf{H}^{2}$, i.e., $\omega^{1}=(1,0)^{*}, \omega^{2}=(i, 0)^{*}, \ldots, \omega^{8}=(0, k)^{*}$. Since

$$
\omega_{I}(x, y)=\langle x i, y\rangle, \quad \omega_{J}(x, y)=\langle x j, y\rangle, \quad \omega_{K}(x, y)=\langle x k, y\rangle .
$$

$$
\begin{aligned}
& \omega_{I}=\omega^{12}-\omega^{34}+\omega^{56}-\omega^{78} \\
& \omega_{J}=\omega^{13}-\omega^{42}+\omega^{57}-\omega^{86} \\
& \omega_{K}=\omega^{14}-\omega^{23}+\omega^{58}-\omega^{67}
\end{aligned}
$$


Therefore,

$$
\begin{aligned}
\Phi_{K}= & -\frac{1}{2} \omega_{I}^{2}-\frac{1}{2} \omega_{J}^{2}+\frac{1}{2} \omega_{K}^{2} \\
= & \omega^{1234}+\omega^{5678}-\omega^{1256}+\omega^{1278}+\omega^{3456}-\omega^{3478} \\
& -\omega^{1357}+\omega^{1386}-\omega^{2457}-\omega^{2468}+\omega^{1458}-\omega^{1467}-\omega^{2358}+\omega^{2367} .
\end{aligned}
$$

Now, let $\tilde{\omega}^{1}, \ldots, \tilde{\omega}^{8}$ denote the standard dual basis for $O$, dual to the basis:

$$
1, i, j, k, e, i e, j e, k e, \text { for } O \text {. }
$$

The Cayley calibration is given by (see [HL, p. 120]):

$$
\begin{aligned}
\Phi= & \tilde{\omega}^{1234}+\tilde{\omega}^{5678}+\tilde{\omega}^{1256}-\tilde{\omega}^{1278}-\tilde{\omega}^{3456}+\tilde{\omega}^{3478} \\
& +\tilde{\omega}^{1357}-\tilde{\omega}^{1386}+\tilde{\omega}^{2457}+\tilde{\omega}^{2468}+\tilde{\omega}^{1458}-\tilde{\omega}^{1467}-\tilde{\omega}^{2358}+\tilde{\omega}^{2367} .
\end{aligned}
$$

Finally note that the map $L: \mathbf{H}^{2} \rightarrow \mathbf{O}$ pulls back $\tilde{\omega}^{j}$ to $\omega^{j}$ for $j=$ $1,2,3,4,5$, and 8 ; and pulls back $\tilde{\omega}^{j}$ to $-\omega^{j}$ for $j=6$ and 7. Comparing (2.23) and (2.24) the lemma follows.

Because of Lemma 2.21, the proof that $H=\operatorname{Spin}(7)$, when $n=2$ (see (2.15)), is an immediate consequence of the fact that the subgroup $H^{\prime} \subset \mathrm{SO}(8)$ which fixes $\Phi$ is $\operatorname{Spin}(7)$. First, the fact that $H^{\prime}$ contains $\operatorname{Spin}(7)$ follows, as noted in [HL], since $\operatorname{Spin}(7)$ is the subgroup of isometries of $\mathbf{O}$ generated by $\left\{R_{u}: u \in \operatorname{Im} \mathbf{O},|u|=1\right\}$ and since $R_{u}^{*}(\Phi)=\Phi$. It is well known that $\operatorname{Spin}(7)$ is a maximal closed subgroup of $\operatorname{SO}(8)$. Since $\Phi$ is not fixed by $\mathrm{SO}(8)$, this proves that $H^{\prime}=\operatorname{Spin}(7)$ and completes the proof of Lemma 2.14.

Lemma 2.21 combined with (2.19) yields

Corollary 2.25. Suppose $V$ is a quaternionic 2-plane in $\mathbf{H}^{n}$. Then $\left.\Phi_{K}\right|_{V}$ is a calibration. That is,

$$
\Phi_{K}(\xi) \leq|\xi| \text { for all } \xi \in G_{\mathbf{R}}(4, V) .
$$

Proof. Since $\Phi_{K}$ is fixed by $\operatorname{Sp}(n)$, we may assume that $V=\mathbf{H}^{2} \times\{0\}$ and then apply (2.19).

Definition 2.26. Given a quaternionic 2-plane $V \subset \mathbf{H}^{n}$, let $\mathrm{CAY}(V)$ denote the set of oriented real 4-planes $\xi \in G_{\mathbf{R}}(4, V)$ in $V$ for which equality $\Phi_{K}(\xi)=|\xi|$ holds.

Now we can state the main result of this paper.

Theorem 2.27. The fundamental four form

$$
\Phi_{K}-\frac{1}{2} \omega_{I}^{2}-\frac{1}{2} \omega_{J}^{2}+\frac{1}{2} \omega_{K}^{2} \in \Lambda^{4} \mathbf{H}^{n}
$$

is a calibration, i.e.,

$$
\Phi_{K}(\xi) \leq|\xi| \text { for all } \xi \in G_{\mathbf{R}}\left(4, \mathbf{H}^{n}\right)
$$


Moreover, equality, $\Phi_{K}(\xi)=|\xi|$, holds if and only if (2.29)

$\operatorname{span}_{R} \xi \subset V$ for some quaternionic 2-plane $V \subset \mathbf{H}^{n}$, and $\xi \in \mathrm{CAY}(V)$.

In fact, each $\xi$ belonging to the contact set

$$
G\left(\Phi_{K}\right) \equiv\left\{\xi \in G_{\mathbf{R}}\left(4, \mathbf{H}^{n}\right): \Phi_{K}(\xi)=1\right\}
$$

can be put in the following normal form with respect to $\mathrm{Sp}(n) \cdot\left(S^{1} \cup R_{I} S^{1}\right)$.

$$
\xi=\left(c_{1} \varepsilon_{1}+s_{1} \varepsilon_{2}\right) \wedge\left(c_{2} I \varepsilon_{1}+s_{2} I \varepsilon_{2}\right) \wedge\left(c_{2} J \varepsilon_{1}-s_{2} J \varepsilon_{2}\right) \wedge\left(c_{1} K \varepsilon_{1}-s_{1} K \varepsilon_{2}\right)
$$

with angles $\phi_{1}$ and $\phi_{2}$ satisfying

$$
0 \leq \phi_{2} \leq \phi_{1} \leq \pi / 2 \text { and } \phi_{1}+\phi_{2} \leq \pi / 2 \text {. }
$$

Here $\varepsilon_{1} \equiv(1,0,0, \ldots, 0), \quad \varepsilon_{2} \equiv(0,1,0, \ldots, 0)$, and $\left(c_{i}, s_{i}\right)$ denotes $\left(\cos \phi_{i}, \sin \phi_{i}\right), i=1,2$.

Remark 2.33. Let $u_{1} \equiv c_{1} \varepsilon_{1}+s_{1} \varepsilon_{2}, u_{1} \equiv c_{2} I \varepsilon_{1}+s_{2} I \varepsilon_{2}, u_{3} \equiv c_{2} J \varepsilon_{1}-s_{2} J \varepsilon_{2}$, and $u_{4}=c_{1} K \varepsilon_{1}-s_{1} K \varepsilon_{2}$, so that $\xi$ has normal form $\xi \equiv u_{1} \wedge u_{2} \wedge u_{3} \wedge u_{4}$. The angles $\phi_{1}$ and $\phi_{2}$ can be obtained from the eigenvalues of $\omega_{K}$ restricted to $\operatorname{span} \xi$. In fact

$$
\begin{aligned}
& \left.\omega_{I}\right|_{\text {span } \xi}=\cos \left(\phi_{1}-\phi_{2}\right)\left(u_{1} \wedge u_{2}-u_{3} \wedge u_{4}\right), \\
& \left.\omega_{J}\right|_{\text {span } \xi}=\cos \left(\phi_{1}+\phi_{2}\right)\left(u_{1} \wedge u_{3}+u_{2} \wedge u_{4}\right), \\
& \left.\omega_{K}\right|_{\text {span } \xi}=\cos \left(2 \phi_{1}\right) u_{1} \wedge u_{4}-\cos \left(2 \phi_{2}\right) u_{2} \wedge u_{3} .
\end{aligned}
$$

In order to prove Theorem 2.27 we need a more convenient expression for $\Phi_{K}$ using the complex structure $I$ on $\mathbf{H}^{n}$ (we could just as easily choose any one of the complex structures $\cos \theta I+\sin \theta J$ on $\mathbf{H}^{n}$ ). Since $\mathbf{H} \equiv \mathbf{C} \oplus j \mathbf{C}$, the identification $\mathbf{H}^{n} \equiv \mathbf{C}^{n} \oplus j \mathbf{C}^{n} \cong \mathbf{C}^{2 n}$ provides a complex linear isomorphism $\mathbf{H}^{n} \cong \mathbf{C}^{2 n}$, where $\mathbf{H}^{n}$ is equipped with the complex structure $I$. Now each $p, q \in \mathbf{H}^{n}$ can be uniquely expressed as

$$
p=z+j w, \quad q=\xi+j \eta
$$

with $z, w, \xi, \eta \in \mathbf{C}^{n}$.

Using these complex coordinates, the quaternionic hermitian symmetric bilinear form $\varepsilon(p, q) \equiv \bar{p} q$ can be expressed as

$$
\bar{p} q=\bar{z} \xi+\bar{w} \eta+j(z \eta-w \xi) .
$$

(Recall that $\bar{p} q$ denotes $\sum_{j=1}^{n} \bar{p}^{j} q^{j}$ and let $z \eta$ denote $\sum_{j=1}^{n} z^{j} \eta^{j}$, etc.) Let $H(p, q) \equiv \bar{z} \xi+\bar{w} \eta$ denote the standard $\mathbf{C}$-hermitian symmetric bilinear form on $\mathbf{C}^{2 n}$, and let $\sigma(p, q) \equiv z \eta-w \xi$ denote the standard $\mathbf{C}$-skew bilinear form (also called the standard $\mathbf{C}$-symplectic form) on $\mathbf{C}^{2 n}$. Note that

$$
\sigma=d z^{1} \wedge d w^{1}+\cdots+d z^{n} \wedge d w^{n} .
$$

(2.34) can be reexpressed as

$$
\varepsilon=H+j \sigma
$$


Comparing $\left(2.34^{\prime}\right)$ with $(2.3)$ yields

$$
H=\langle,\rangle+i \omega_{I} \text { and } \sigma=\omega_{J}-i \omega_{K} .
$$

Now using this choice of the complex structure $I$ on $H^{n}$ the fundamental 4-form

can also be expressed as

$$
\Phi_{K} \equiv-\frac{1}{2} \omega_{I}^{2}-\frac{1}{2} \omega_{J}^{2}+\frac{1}{2} \omega_{K}^{2}
$$

$$
\Phi_{K}=-\frac{1}{2} \omega_{I}^{2}-\operatorname{Re} \frac{1}{2} \sigma^{2}
$$

The proof that $\Phi_{K}$ is a calibration (Theorem 2.27) depends on the fact that $\operatorname{Re} \frac{1}{2} \sigma^{2}$ is a calibration.

Theorem 2.38 The 4-form $\operatorname{Re} \frac{1}{2} \sigma^{2} \in \Lambda^{4} \mathrm{C}^{2 n}$ is a calibration, i.e.,

$$
\operatorname{Re} \frac{1}{2} \sigma^{2}(\eta) \leq|\eta| \text { for all } \eta \in G_{\mathbf{R}}\left(4, C^{2 n}\right) \text {. }
$$

Moreover, if equality holds then there exists a quaternionic 2-plane $V \subset \mathbf{C}^{2 n} \cong$ $\mathbf{H}^{n}$ with $\operatorname{span} \eta \subset V$.

Remark 2.40. If $V \equiv \mathbf{H}^{2} \times\{0\} \subset \mathbf{H}^{\prime \prime}$ then $\frac{1}{2} \sigma^{2}=\frac{1}{2}\left(d z^{1} \wedge d w^{1}+d z^{2} \wedge d w^{2}\right)^{2}=$ $d z^{1} \wedge d w^{1} \wedge d z^{2} \wedge d w^{2}$ is a unit complex volume form for $\mathbf{C}^{4} \cong V$. Thus $\left.\operatorname{Re} \frac{1}{2} \sigma^{2}\right|_{V}=\operatorname{Re}\left(d z^{1} \wedge d w^{1} \wedge d z^{2} \wedge d w^{2}\right)$ is a calibration called the special Lagrangian calibration (see [HL] for details). Similarly, $\left.\operatorname{Re} \frac{1}{2} \sigma^{2}\right|_{V}$ is a special Lagrangian calibration for any quaternionic two plane $V \subset \mathbf{H}^{n}$.

Let $\operatorname{SLAG}(V)$ denote the set $\left\{\eta \in G_{\mathbf{R}}(4, V): \operatorname{Re} \frac{1}{2} \sigma^{2}(\eta)=1\right\}$, where $\left.\operatorname{Re} \frac{1}{2} \sigma^{2}\right|_{V}$ attains its maximum value, 1. Thus the second half of Theorem 2.38 may be restated as

$$
\begin{array}{cl}
\operatorname{Re} \frac{1}{2} \sigma^{2}(\eta)=|\eta| & \text { if and only if } \eta \in \operatorname{SLAG}(V) \text { for some } \\
& \text { quaternionic 2-plane } V \subset \mathbf{H}^{n} .
\end{array}
$$

\section{THE PROOF OF THE MAIN RESULT}

This section contains the proofs of the two theorems stated in $\S 2$.

Proof of Theorem 2.38. This theorem says that $\operatorname{Re} \frac{1}{2} \sigma^{2}$ is a calibration with contact set

$$
G\left(\operatorname{Re} \frac{1}{2} \sigma^{2}\right)=\bigcup_{V \in G_{H}\left(2, \mathrm{H}^{n}\right)} \operatorname{SLAG}(V) .
$$

First note that $\frac{1}{2} \sigma^{2}$ has bidegree 4,0 (with respect to the complex structure I). Therefore,

$$
\left(\frac{1}{2} \sigma^{2}\right)(\xi)=0 \text { if } \xi \in G_{R}\left(4, \mathbf{H}^{n}\right) \text { contains a complex line . }
$$

To prove (3.2) assume that $\xi \equiv e \wedge I e \wedge u \wedge v$ contains the complex line $\operatorname{span}_{\mathbf{R}}\{e, I e\}$. Then $e \wedge I e$ has bidegree 1,1 , while $u \wedge v$ can be expressed 
as the sum of terms of bidegree $2,0,1,1$, and 0,2 . Therefore $\xi$ has no bidegree 4,0 component.

Each $\eta \in G_{\mathbf{R}}\left(4, \mathbf{C}^{2 n}\right)$ can be put in the following canonical form (see [HL, p. 70])

$$
\eta \equiv e_{1} \wedge\left(\cos \theta_{1} I e_{1}+\sin \theta_{1} e_{2}\right) \wedge e_{3} \wedge\left(\cos \theta_{2} I e_{3}+\sin \theta_{2} e_{4}\right)
$$

where $e_{1}, e_{2}, e_{3}, e_{4}$ is part of a unitary basis for $\mathbf{C}^{2 n}$ (with complex structure I), and the angles satisfy $0 \leq \theta_{1} \leq \pi / 2, \theta_{1} \leq \theta_{2} \leq \pi$. Because of (3.2)

$$
\operatorname{Re} \frac{1}{2} \sigma^{2}(\eta)=\operatorname{Re} \frac{1}{2} \sigma^{2}\left(e_{1} \wedge e_{2} \wedge e_{3} \wedge e_{4} \wedge\right) \sin \theta_{1} \sin \theta_{2} .
$$

Thus $\operatorname{Re} \frac{1}{2} \sigma^{2}$ attains its maximum value when $\eta=e_{1} \wedge e_{2} \wedge e_{3} \wedge e_{4}$ is I-isotropic. That is,

$$
\varepsilon \in \operatorname{span} \eta \text { implies } I \varepsilon \perp \operatorname{span} \eta \text {. }
$$

Now assume that $\operatorname{Re} \frac{1}{2} \sigma^{2}$ attains its maximum. Choose a unit vector $\varepsilon_{1} \in$ $\operatorname{span} \eta$. Then $\eta=\varepsilon_{1} \wedge \tau$ where $\tau$ is an $I$-isotropic 3 -vector in $\left\{\varepsilon_{1}, I \varepsilon_{1}\right\}^{\perp}$. Completing $\varepsilon_{1}$ to a quaternionic orthonormal basis $\varepsilon_{1}, \varepsilon_{2}, \ldots, \varepsilon_{n}$, the subspace $\left\{\varepsilon_{1}, I \varepsilon_{1}\right\}^{\perp}$ may be expressed as $j \mathbf{C} \oplus \mathbf{H}^{n-1}$. Thus $\operatorname{dim}_{\mathbf{R}}(\operatorname{span} \tau) \cap\left(\{0\} \times \mathbf{H}^{n-1}\right) \geq$ 1. That is, $\varepsilon_{2}$ may be chosen in $\operatorname{span} \tau$. Hence

$$
\eta=\varepsilon_{1} \wedge \varepsilon_{2} \wedge \beta
$$

with $\beta \in G_{2}\left(j \mathbf{C} \oplus j \mathbf{C} \oplus \mathbf{H}^{n-2}\right)$ an $I$-isotropic 2-plane.

Therefore,

$$
\begin{aligned}
\operatorname{Re} \frac{1}{2} \sigma^{2}(\eta) & \left.\left.=\operatorname{Re} \frac{1}{2} \sigma^{2}\left(\varepsilon_{1} \wedge e_{2} \wedge \beta\right)=\left(\varepsilon_{2}\right\lrcorner\left(\varepsilon_{1}\right\lrcorner \operatorname{Re} \frac{1}{2} \sigma^{2}\right)\right)(\beta) \\
& \left.\left.\left.\left.=\left(\varepsilon_{2}\right\lrcorner\left(\varepsilon_{1}\right\lrcorner \frac{1}{2} \omega_{J}^{2}\right)\right)(\beta)-\left(\varepsilon_{2}\right\lrcorner\left(\varepsilon_{1}\right\lrcorner \frac{1}{2} \omega_{K}^{2}\right)\right)(\beta) .
\end{aligned}
$$

Now, recalling the coordinate formulas (2.22) for $\omega_{J}$ and $\omega_{K}$ on the quaternionic axis 2-plane $\mathbf{H}^{2}$ (which we now take to be the $\mathbf{H}$-span of $\varepsilon_{1}$ and $\varepsilon_{2}$ );

$$
\begin{aligned}
& \frac{1}{2} \omega_{J}^{2}=-\omega^{1234}+\omega^{1357}+\omega^{1368}+\omega^{2457}+\omega^{2468}-\omega^{5678} \\
& \frac{1}{2} \omega_{K}^{2}=-\omega^{1234}+\omega^{1458}-\omega^{1467}-\omega^{2358}+\omega^{2367}-\omega^{5678} .
\end{aligned}
$$

Since $\varepsilon_{1}^{*}=\omega^{1}$ and $\varepsilon_{2}^{*}=\omega^{5}$,

$$
\left.\left(\varepsilon_{2}\right\lrcorner\left(\varepsilon_{1}-\frac{1}{2} \omega_{J}^{2}\right)\right)(\beta)=-\omega^{37}(\beta),
$$

and

$$
\left.\left.\left(\varepsilon_{2}\right\lrcorner\left(\varepsilon_{1}\right\lrcorner \frac{1}{2} \omega_{K}^{2}\right)\right)(\beta)=-\omega^{48}(\beta)
$$

Therefore,

$$
\operatorname{Re} \frac{1}{2} \sigma^{2}\left(\varepsilon_{1} \wedge \varepsilon_{2} \wedge \beta\right)=\left(-\omega^{37}+\omega^{48}\right)(\beta)
$$

It is a classical result that the two form $-\omega^{37}+\omega^{48}$ is a calibration, i.e.,

$$
\left(-\omega^{37}+\omega^{48}\right)(\beta) \leq 1
$$


This proves that $\operatorname{Re} \frac{1}{2} \sigma^{2}$ is a calibration. Moreover, if equality occurs in (3.10) then $\operatorname{span} \beta$ is contained in the four dimensional space spanned by the third, fourth, seventh, and eighth coordinate axes in $\mathbf{H}^{2}=\mathbf{R}^{8}$. Therefore $\operatorname{span} \eta=\operatorname{span} \varepsilon_{1} \wedge \varepsilon_{1} \wedge \beta$ is contained in $\mathbf{H}^{2}$, completing the proof of Theorem 2.38 .

Proof of Main Theorem 2.27. Each $-\xi \in G_{\mathbf{R}}\left(4, \mathbf{C}^{2 n}\right)$ can be put in the following canonical form (3.3):

$$
\begin{aligned}
-\xi \equiv & e_{1} \wedge\left(\cos \theta_{1} I e_{1}+\sin \theta_{1} e_{2}\right) \wedge e_{3} \wedge\left(\cos \theta_{2} I e_{3}+\sin \theta_{2} e_{4}\right) \\
= & -\cos \theta_{1} \cos \theta_{2} e_{1} \wedge I e_{1} \wedge e_{3} \wedge I e_{3}-\cos \theta_{1} \sin \theta_{2} e_{1} \wedge I e_{1} \wedge e_{3} \wedge e_{4} \\
& -\sin \theta_{1} \cos \theta_{2} e_{1} \wedge e_{2} \wedge e_{3} \wedge I e_{3}-\sin \theta_{1} \sin \theta_{2} e_{1} \wedge e_{2} \wedge e_{3} \wedge e_{4} .
\end{aligned}
$$

Recall the formula (2.37) $\Phi_{K} \equiv-\frac{1}{2} \omega_{I}^{2}-\frac{1}{2} \operatorname{Re} \sigma^{2}$, for $\Phi_{K}$. First, note $-\frac{1}{2} \omega_{I}^{2}(\xi)$ $=\cos \theta_{1} \cos \theta_{2}$. Second, note that

$$
-\frac{1}{2} \sigma^{2}(\xi)=\sin \theta_{1} \sin \theta_{2} \frac{1}{2} \sigma^{2}\left(e_{1} \wedge e_{2} \wedge e_{3} \wedge e_{4}\right)
$$

because of (3.2). Therefore,

$$
\Phi_{K}(\xi)=\cos \theta_{1} \cos \theta_{2}+A \sin \theta_{1} \sin \theta_{2},
$$

where

$$
A \equiv \operatorname{Re} \frac{1}{2} \sigma^{2}\left(e_{1} \wedge e_{2} \wedge e_{3} \wedge e_{4}\right) .
$$

Because of Theorem 2.38,

$$
|A| \leq 1 \text { and }|A|=1
$$

if and only if $\operatorname{span}\left\{e_{1}, e_{2}, e_{3}, e_{4}\right\} \subset V$ for some quaternionic plane $V \subset \mathbf{H}^{n}$.

This proves that $\Phi_{K}$ is a calibration, i.e.,

$$
\Phi_{K}(\xi) \leq 1
$$

Moreover, if $\Phi_{K}(\xi)=1$ then $|A|=1$ so that $\operatorname{span}\left\{e_{1}, e_{2}, e_{3}, e_{4}\right\} \subset V$ for some quaternionic plane $V \subset \mathbf{H}^{n}$. Since the real span, span $\xi$, is contained in the $H$-span of $\left\{e_{1}, e_{2}, e_{3}, e_{4}\right\}$ it follows that span $\xi \subset V$, verifying (2.29).

It remains to prove the normal form (2.30) for $\mathrm{Sp}(n) \cdot\left(S^{1} \cup R_{I} S^{1}\right)$ acting on the contact set $G\left(\Phi_{K}\right)$. Because of (2.29), the general case $n \geq 2$ can be reduced to the special case $n=2$. Given $\xi \in G\left(\Phi_{K}\right)$, let $\xi \mathbf{H}$ denote the quaternionic span of the vectors in $\xi$. By (2.29), $\operatorname{dim}_{H} \xi \mathbf{H}$ is either 1 or 2 . If $\operatorname{dim}_{H} \boldsymbol{\xi} \mathbf{H}=1$ then $\operatorname{span} \xi$ is a quaternionic line in $\mathbf{H}^{n}$. Conversely, all quaternionic lines in $\mathbf{H}^{n}$ belong to $G\left(\Phi_{K}\right)$, i.e., $P^{n-1}(\mathbf{H}) \subset G\left(\Phi_{K}\right)$. Now suppose $\operatorname{dim}_{H} \xi \mathbf{H}=2$. Since $\operatorname{Sp}(n)$ acts transitively on the quaterionic 2planes in $H^{n}$, we may assume that $\xi \mathbf{H} \equiv \mathbf{H}^{2} \times\{0\} \subset \mathbf{H}^{n}$, the $\mathbf{H}$-span of the first two axis vectors in $\mathbf{H}^{n}$. The stabilizer of $\mathbf{H}^{2} \times\{0\}$ in $\operatorname{Sp}(n) \cdot\left(S^{1} \cup R_{l} S^{1}\right)$ is the subgroup

$$
H^{\prime}=(\operatorname{Sp}(2) \times \operatorname{Sp}(n-2)) \cdot\left(S^{1} \cup R_{I} S^{1}\right) .
$$


It follows that the normal forms of $\xi \in G\left(\Phi_{K}\right)$, under $\operatorname{Sp}(n) \cdot\left(S^{1} \cup R_{I} S^{1}\right)$, are identical with the normal forms of $\xi \in \operatorname{CAY}\left(H^{n}\right)$ under the group

$$
\mathrm{Sp}(2) \cdot\left(S^{1} \cup R_{I} S^{1}\right) \text {. }
$$

Note that this is a proper subgroup of the full automorphism group, $\operatorname{Spin}(7)$, of $\Phi_{K}$ when $n=2$.

To give the proof of the normal form (2.30) when $n=2$, it is convenient now to use the map $L: \mathbf{H}^{2} \rightarrow \mathbf{O}$ to transfer our problem over to $\mathbf{O}$. First, we identify the image of $\mathrm{Sp}(2) \cdot\{1\}$ in $\operatorname{Spin}(7) \subseteq \mathrm{SO}(\mathbf{O})=\mathrm{SO}(8)$. Recall that $R_{i}^{\mathbf{O}}, R_{j}^{\mathbf{O}} \in \operatorname{Spin}(7)$. Clearly $\mathrm{Sp}(2)$ is identified as the subgroup of $\operatorname{Spin}(7)$ which commutes with $R_{i}$ and $R_{j}$. Thus, we call this subgroup $\operatorname{Sp}(2)$ also. The group $\{1\} \cdot S^{1}$ clearly transfers over to the group, also denoted $S^{1}$, given by $\left\{e^{t R_{i} \circ R_{1}} \mid t \in \mathbf{R}\right\}$. (Remember: $R_{j}^{\mathbf{O}} \circ R_{i}^{\mathbf{O}} \neq R_{k}^{\mathbf{O}}$ !) Now $L$ can be used to identify $\mathrm{CAY}\left(H^{2}\right)$ with $\mathrm{CAY}$.

As noted in $\S 2, \operatorname{Spin}(7)$ acts transitively on CAY. The stabilizer $U$ in $\operatorname{Spin}(7)$ of $\xi_{0} \equiv 1 \wedge i \wedge j \wedge k \in \mathrm{CAY}$ can be described as follows: Let $\operatorname{Sp}(1) \times \operatorname{Sp}(1) \times \operatorname{Sp}(1)$ act on $\mathbf{O}$ by $\left(u_{1}, u_{2}, u_{3}\right) \cdot(a+b e)=u_{3} a \bar{u}_{1}+\left(u_{2} b \bar{u}_{1}\right) e$. This action preserves $\Phi$ and is almost faithful, the kernel being given by $Z_{2}=\{ \pm(1,1,1)\}$. Let $U=(\operatorname{Sp}(1) \times \operatorname{Sp}(1) \times \operatorname{Sp}(1)) / Z_{2}$ denote the image subgroup in $\operatorname{Spin}(7)$. It is not difficult to see that $U$ is the stabilizer of $\xi_{0}$ in $\operatorname{Spin}(7)$. That is

$$
\mathrm{CAY} \cong \operatorname{Spin}(7) / U
$$

with $U \equiv(\mathrm{Sp}(1) \times \mathrm{Sp}(1) \times \mathrm{Sp}(1)) / \mathrm{Z}_{2}$ (see $\left.[\mathrm{HL}]\right)$.

We can get a more familiar description of CAY as a homogeneous space by noting that $U$ contains the center of $\operatorname{Spin}(7)$, namely $Z=\left\{ \pm I_{8}\right\}$. Thus, $\operatorname{Spin}(7) / U \simeq \operatorname{Spin}(7) / Z) /(U / Z)$. Now $\operatorname{Spin}(7) / Z=\operatorname{SO}(7)$, and $\bar{U} \equiv U / Z=$ $\mathrm{SO}(3) \times \mathrm{SO}(4)$. Thus we have an isomorphism

$$
\mathrm{CAY} \cong \mathrm{SO}(7) / \mathrm{SO}(3) \times \mathrm{SO}(4) \cong G\left(3, \mathbf{R}^{7}\right)
$$

where $G\left(3, \mathbf{R}^{7}\right)$ is the Grassmanian of oriented 3-planes in $\mathbf{R}^{7}$.

It will be useful to have a more explicit description of the diffeomorphism $\mathrm{CAY}=G\left(3, \mathbf{R}^{7}\right)$. If $\xi \in \mathrm{CAY}$ is written in the form $\xi=e_{1} \wedge e_{2} \wedge e_{3} \wedge e_{4}$ where $e_{i}$ are orthonormal, then set $\tilde{\xi}=\left(e_{2} \times e_{1}\right) \wedge\left(e_{3} \times e_{1}\right) \wedge\left(e_{4} \times e_{1}\right) \in \Lambda^{3}(\operatorname{Im} \mathbf{O})$. (Recall that, for $x, y \in \mathbf{O}, x \times y=\frac{1}{2}(\bar{y} x-\bar{x} y)$ is an element of $\operatorname{Im} \mathbf{O} \simeq \mathbf{R}^{7}$. If $x, y$ are orthonormal, then $x \times y$ is a unit imaginary octonian.) Using the properties proved in [HL, §IV], one sees that the mapping $\xi \rightarrow \tilde{\xi}$ establishes a well-defined diffeomorphism $\mathrm{CAY} \stackrel{\sim}{\rightarrow} G(3, \operatorname{Im} \mathbf{O})=G\left(3, \mathbf{R}^{7}\right)$. In particular, note that if $\xi \in \mathrm{CAY}$ and $\xi^{\perp}$ denotes the orthogonal 4-plane endowed with the appropriate orientation so that $\xi^{\perp} \in \mathrm{CAY}$, then $\left(\xi^{\perp}\right)^{\sim}=-\tilde{\xi}$. As a particular example, $(1 \wedge i \wedge j \wedge k)^{\sim}=i \wedge j \wedge k$ and $(e \wedge i e \wedge j e \wedge k e)^{\sim}$ equals $-i \wedge j \wedge k$. As shown in [HL], there is a homomorphism $\chi: \operatorname{Spin}(7) \rightarrow \mathrm{SO}(7)=\mathrm{SO}(\operatorname{Im} \mathbf{O})$ given by $\chi(g)(w)=g\left(g^{-1}(1) w\right)$ for $g \in \operatorname{Spin}(7)$ and $w \in \operatorname{Im} \mathbf{O}$. This homomorphism 
satisfies $g x \times g y=\chi(g)(x \times y)$, so it easily follows that $\chi(U)=\bar{U}=$ the stabilizer of $i \wedge j \wedge k$ where, as previously defined, $U \subseteq \operatorname{Spin}(7)$ is the stabilizer of $\xi_{0}=1 \wedge i \wedge j \wedge k$. Note that $\chi$ is a 2-1 covering with kernel $\{ \pm 1\}$, the center of $\operatorname{Spin}(7)$. It is now easy to compute that $\chi\left(\operatorname{Sp}(2) \cdot\left(S^{1} \cup R_{i} S^{1}\right)\right)=$ $S(\mathrm{O}(2) \times \mathrm{O}(5))$. This is the subgroup of $\mathrm{SO}(7) \quad(=\mathrm{SO}(\operatorname{Im} \mathrm{O})$ ) which preserves the 2-plane spanned by $\{i, j\} \subseteq \operatorname{Im} \mathbf{O}$. Our problem is now reduced to finding a normal form for oriented 3-planes in $\operatorname{Im} \mathbf{O}$ under the subgroup of $\mathrm{SO}(\operatorname{Im} \mathbf{O})$ which fixes the 2-plane $\{i, j\}$. This problem is easily solved. For any pair of angles $\left(\theta_{1}, \theta_{2}\right)$, consider the 3-plane

$$
\eta_{\theta}=\left[\left(\cos \theta_{1}\right) i+\left(\sin \theta_{1}\right) i e\right] \wedge\left[\left(\cos \theta_{2}\right) j+\left(\sin \theta_{2}\right) j e\right] \wedge k .
$$

It is easy to see that any oriented 3-plane $\eta$ is equivalent to $\eta_{\theta}$ under the action of $S(\mathrm{O}(2) \times \mathrm{O}(5))$ for some $\theta=\left(\theta_{1}, \theta_{2}\right)$. Moreover, we may assume $0 \leq \theta_{1} \leq \theta_{2} \leq \pi / 2$ (this makes $\left(\theta_{1}, \theta_{2}\right)$ unique). Note that in the generic case, $0<\theta_{1}<\pi / 2$, the stabilizer of $\eta_{\theta}$ in $S(\mathrm{O}(2) \times \mathrm{O}(5))$ is a subgroup of dimension 1 with four components. It may be described as the set of transformations of the form

$$
\left(\begin{array}{c}
i \\
j \\
k \\
e \\
i e \\
j e \\
k e
\end{array}\right) \rightarrow\left(\begin{array}{c}
\varepsilon_{1} i \\
\varepsilon_{2} j \\
\varepsilon_{1} \varepsilon_{2} k \\
\varepsilon_{1}(i e) \\
\varepsilon_{2}(j e) \\
(\cos \phi) e+(\sin \phi) k e \\
\varepsilon_{1} \varepsilon_{2}((-\sin \phi) e+(\cos \phi) k e)
\end{array}\right)
$$

where $\varepsilon_{1}^{2}=\varepsilon_{2}^{2}=1$, and $\phi$ is an arbitrary angle. We will examine the nongeneric cases further on.

Let $\phi_{1}, \phi_{2}, \phi_{3}, \phi_{4}$ be four angles and set $\left(c_{i}, s_{i}\right)=\left(\cos \phi_{i}, \sin \phi_{i}\right)$. Write $\xi_{\phi}=\left(c_{1} l+s_{1} e\right) \wedge\left(c_{2} i+s_{2} i e\right) \wedge\left(c_{3} j+s_{3} j e\right) \wedge\left(c_{4} k+s_{4} k e\right)$. Using the formula (2.24) for $\Phi$ we have

$$
\Phi\left(\xi_{\phi}\right)=\cos \left(\phi_{1}+\phi_{2}+\phi_{3}+\phi_{4}\right) .
$$

Thus, $\xi_{\phi} \in \mathrm{CAY}$ iff $\phi_{1}+\phi_{2}+\phi_{3}+\phi_{4} \equiv 0 \bmod 2 \pi$. In this case, it is easy to compute that

$$
\tilde{\xi}_{\phi}=\left(\tilde{c}_{2} i+\tilde{s}_{2} i e\right) \wedge\left(\tilde{c}_{3} j+\tilde{s}_{3} j e\right) \wedge\left(\tilde{c}_{4} k+\tilde{s}_{4} k e\right)
$$

where $\left(\tilde{c}_{i}, \tilde{s}_{i}\right)=\left(\cos \left(\phi_{i}+\phi_{1}\right), \sin \left(\phi_{i}+\phi_{1}\right)\right)$ for $i=2,3,4$.

It follows that if we set $\phi=\left(\frac{1}{2}\left(\theta_{1}+\theta_{2}\right), \frac{1}{2}\left(\theta_{1}-\theta_{2}\right), \frac{1}{2}\left(\theta_{2}-\theta_{1}\right),-\frac{1}{2}\left(\theta_{1}+\theta_{2}\right)\right)$ then $\xi_{\phi} \in \mathrm{CAY}$ and $\tilde{\xi}_{\phi}=\eta_{\theta}$. By translating this information back into $\mathbf{H}^{2}$ via the map $L$, the normal form (2.31) is verified.

Remark 3.21. Note that the given inequalities describe a triangle in the $\phi$-plane. See Figure 1.

It is worth remarking on the special nature of some of the vertices and edges of this triangle. 


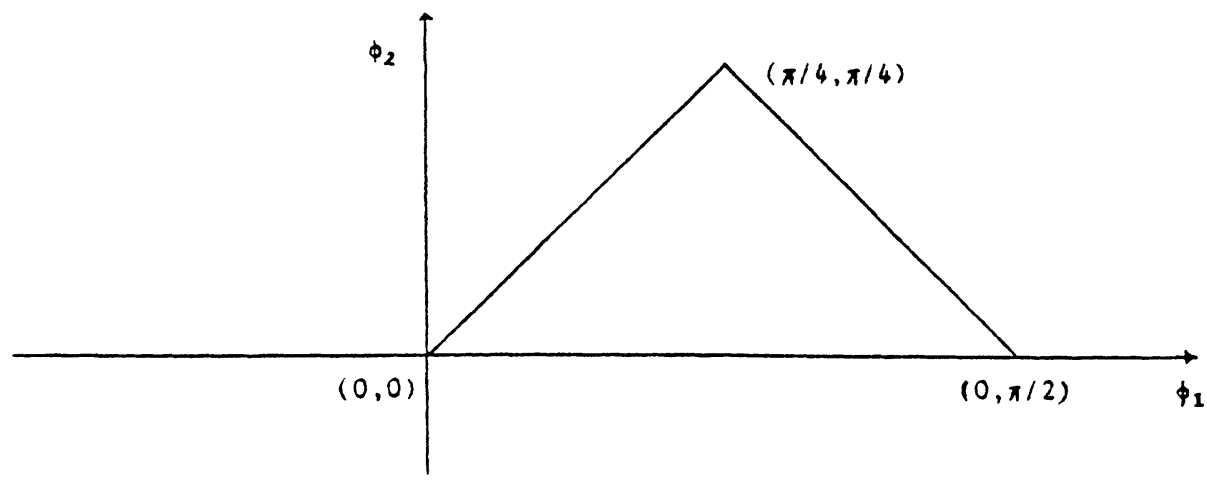

FIGURE 1

The case where $\phi_{1}=\phi_{2}=\phi \subset[0, \pi / 4]$ consists of those $\xi \in G\left(\Phi_{K}\right)$ which are anticomplex with respect to some complex structure $I_{\theta}=(\cos \theta) I+(\sin \theta) J$. Here, "anticomplex" means that $-\xi$, with its natural orientation, is an $I_{\theta}$ complex 2-plane in $\mathbf{H}^{n}$ (endowed with the complex structure $I_{\theta}$ ). To see this, note that, under $\mathbf{H}^{n}$, all $I_{\theta}$ are equivalent and that $\xi^{\prime}$ as described in the above theorem is $I$-complex when $\phi_{1}=\phi_{2}$. Conversely, as was already noted in $\S 2$, all $I$-complex 2-planes in $\mathbf{H}$ belong to $G\left(\Phi_{K}\right)$ when the orientation is reversed.

The even more special case $\phi_{1}=\phi_{2}=0$ gives the plane $\xi^{\prime}=e_{1} \wedge e_{2} \wedge e_{3} \wedge e_{4}$, a quaternion line in $\mathbf{H}^{n}$.

The edge given by $\phi_{1}+\phi_{2}=\pi / 2,0 \leq \phi_{2} \leq \pi / 4$ consists of 4-planes which are special isotropic with respect to $J$. It follows that a $\xi \in G\left(\Phi_{K}\right)$ which is equivalent to a $\xi^{\prime}$ with $\left(\phi_{1}, \phi_{2}\right)$ on this edge is special isotropic for some calibration of the form $-\operatorname{Re}\left(\frac{1}{2} \sigma_{\theta}^{2}\right)$ where $\sigma_{\theta}=(-\sin \theta) \omega_{I}+(\cos \theta) \omega_{J}-i \omega_{K}$. Note that $\sigma_{\theta}$ is a complex $(2,0)$-form with respect to the complex structure $I_{\theta}$.

The vertex $\left(\phi_{1}, \phi_{2}\right)=(\pi / 2,0)$ consists of those 4-planes in $H^{n}$ which are $K$-complex and isotropic with respect to the form $\sigma=\omega_{I}-\sqrt{-1} \omega_{J}$. This form $\sigma$ is of type $(2,0)$ if we regard $\mathbf{H}^{n}$ as a complex vector space via $K$. Conversely, it is easy to see that a $K$-complex 2-plane lies in $G\left(\Phi_{K}\right)$ iff it is $\sigma$-isotropic.

As a final remark, let us note that, for $n>2, G\left(\Phi_{K}\right)$ is not a smooth submanifold of $G\left(4, H^{n}\right)$. In fact, we have

$$
G\left(\Phi_{K}\right)=\bigcup_{V \in G_{H}\left(2, H^{n}\right)} \operatorname{CAY}(V)
$$


If we let $\operatorname{CAY}^{*}(V)=\mathrm{CAY}(V) \backslash \mathbf{H P}(V)$ denote the set of $\xi \in \mathrm{CAY}(V)$ which are not quaternion lines, then the smooth part of $G\left(\Phi_{K}\right)$ can be written

$$
G^{\cdot}\left(\Phi_{K}\right)=\bigcup_{V \in G_{H}\left(2, H^{\prime \prime}\right)} \operatorname{CAY}^{*}(V)
$$

The singular part is, of course, $\mathbf{H P}^{n-1} \subseteq G\left(4, \mathbf{H}^{n}\right)$.

\section{Maximality}

A calibration $\phi \in \Lambda^{p}\left(\mathbf{R}^{n}\right)^{*}$ determines its contact set

$$
G(\phi) \equiv\left\{\xi \in G\left(p, \mathbf{R}^{n}\right): \phi(\xi)=1\right\} .
$$

This is the intersection of the hyperplane $\phi=1$ in $\Lambda^{p} \mathbf{R}^{n}$ with the Grassmannian $G\left(p, \mathbf{R}^{n}\right)$. The larger the contact set, the larger the geometry associated with the calibration $\phi$. It is natural to consider the case where $G(\phi)$ is maximal. That is,

$$
\begin{aligned}
& G(\phi) \subset G(\psi) \text { implies } G(\phi)=G(\psi) \text { for each calibration } \psi \in \\
& \Lambda^{p}\left(\mathbf{R}^{n}\right)^{*} .
\end{aligned}
$$

If the stronger condition

$$
G(\phi) \subset G(\psi) \text { implies } \psi=\phi \text { for each calibration } \psi \in \Lambda^{p}\left(\mathbf{R}^{n}\right)^{*}
$$

is satisfied then $\phi$ is said to be an exposed calibration.

This terminology is justified by the following facts (see [HL, II.7] for more details). Let $K$ denote the mass ball, i.e., the convex hull of $G\left(p, \mathbf{R}^{n}\right)$ in $\Lambda^{p} \mathbf{R}^{n}$. Let $K^{*} \equiv\left\{\phi \in \Lambda^{p}\left(\mathbf{R}^{n}\right)^{*}: \phi(\xi) \leq 1\right.$ for all $\left.\xi \in K\right\}$ denote the dual convex body, called the comass ball. Thus $K^{*}$ consists of all the parallel calibrations of degree $p$ on $\mathbf{R}^{n}$. If $G(\phi)$ is maximal then it can be shown that $\phi$ is an extreme point of $K^{*}$. Let $\xi$ denote any fixed point in the interior of the convex hull of $G(\phi)$, then $\mathscr{F}^{*}(\xi) \equiv\left\{\psi \in K^{*}: \psi(\xi)=1\right\}$ (the dual facet of the singleton $\{\xi\} \subset K$ ) is just the set of calibrations $\psi$ satisfying $G(\psi) \supseteq G(\phi)$. Now the condition (4.2) can be restated as $\mathscr{F}^{*}(\xi)=\{\phi\}$, i.e., the singleton $\{\phi\}$ is an exposed point of $K^{*}$, not just an extreme point.

Proposition 4.3. For $n=2$, the calibration $\Phi_{K} \in \Lambda^{4}\left(H^{2}\right)^{*}$ is exposed (and hence maximal). Equivalently, the Cayley calibration $\Phi$ is exposed.

Proof. The proposition is an immediate consequence of the "first cousin principle" (stated and proved below), since inspection of the coordinate formula (2.23) for $\Phi_{K} \in \Lambda^{4}\left(\mathbf{H}^{2}\right)^{*}$ shows that each axis 4-plane in $\mathbf{H}^{2}$ is either one of the terms in (2.23) (i.e., belongs to $G\left(\Phi_{K}\right)$ ) or is a first cousin of one of these special axis 4-planes in $G\left(\Phi_{K}\right)$.

This principle is a simple consequence of elementary calculus. It has been used in virtually all of the recent papers on calibrations, starting with [HL]. We include the proof for the sake of completeness. 
Lemma 4.4 (The First Cousin Principle). If $\psi \in \Lambda^{p}\left(\mathbf{R}^{n}\right)^{*}$ is a calibration which is 1 on $\xi \equiv e_{1} \wedge \cdots \wedge e_{p}$, where $e_{1}, \ldots, e_{n}$ is an orthonormal basis for $\mathbf{R}^{n}$, then $\psi$ vanishes on all the first cousins of $\xi$,

$$
\eta_{j k} \equiv e_{1} \wedge \cdots \wedge \hat{e}_{j} \wedge \cdots \wedge e_{p} \wedge e_{k}
$$

where $1 \leq j \leq p$ and $p<k \leq n$.

Proof. Let $f(\theta) \equiv \psi\left(e_{1} \wedge \cdots \wedge\left(\cos \theta e_{j}+\sin \theta e_{k}\right) \wedge \cdots \wedge e_{p}\right)$. Since $f$ attains its maximum value of one at $\theta=0, f^{\prime}(0)=\psi(\eta)$ must vanish.

Theorem 4.6. The fundamental 4-form $\Phi_{K} \in \Lambda^{4}\left(\mathbf{H}^{n}\right)^{*}$ is an exposed calibration (and hence maximal).

Proof. Suppose $\Psi \in \Lambda^{4}\left(\mathbf{H}^{n}\right)^{*}$ is another calibration which is identically one on the subset $G(\Phi) \subset G_{\mathbf{R}}\left(4, \mathbf{H}^{n}\right)$. In order to prove Theorem 4.6, we must show that the difference $\phi \equiv \Phi_{K}-\Psi$ vanishes.

If $V$ is a quaternionic 2-plane in $\mathbf{H}^{n}$ then $V \cong \mathbf{H}^{2}$ and hence $\phi$, restricted to $V$, vanishes by Proposition 4.3. Thus the Maximality Theorem 4.6 is an immediate consequence of the next lemma.

Lemma 4.7. Let

$A \equiv\left\{\xi \in G_{\mathbf{R}}\left(r, \mathbf{H}^{n}\right)\right.$ : there exists a quaternionic 2-plane $V$ with $\left.\operatorname{span}_{R} \xi \subset V\right\}$.

Then $A$ spans $\Lambda^{4} \mathbf{H}^{n}$.

Proof. First we show that each real 2-plane $\eta$ in $\mathbf{H}^{n}$ can be expressed as a sum,

$$
\eta \equiv \sum a_{j} \wedge I a_{j}+\sum b_{j} \wedge J b_{j}+\sum c_{j} \wedge K c_{j}
$$

of 2-planes which are complex lines for one of the three complex structures $I$, $J$, and $K$.

We may choose an $\mathbf{H}$-unitary orthonormal basis $e_{1}, \ldots, e_{n}$ for $\mathbf{H}^{n}$ with $\eta=e_{1} \wedge\left(\cos \theta u+\sin \theta e_{2}\right)$ where $u$ is in the $H$-span of $e_{1}$. Obviously $e_{1} \wedge u$ can be expressed as a sum of the form (4.8). It remains to consider $e_{1} \wedge e_{2}$. Choosing complex coordinates on $\mathbf{H}^{2} \equiv \operatorname{span}_{H}\left\{e_{1}, e_{2}\right\}$ yields:

$$
\begin{array}{ll}
\left(\partial / \partial z_{l}^{1}\right)=e_{1}+i\left(e_{1} I\right), & \left(\partial / \partial w_{I}^{1}\right)=\left(e_{1} J\right)-i\left(e_{1} K\right), \\
\left(\partial / \partial z_{I}^{2}\right)=e_{2}+i\left(e_{2} I\right), & \left(\partial / \partial w_{I}^{2}\right)=\left(e_{2} J\right)-i\left(e_{2} K\right), \\
\left(\partial / \partial z_{J}^{1}\right)=e_{1}+i\left(e_{1} J\right), & \left(\partial / \partial w_{J}^{1}\right)=\left(e_{1} I\right)+i\left(e_{1} K\right), \\
\left(\partial / \partial z_{J}^{2}\right)=e_{2}+i\left(e_{2} J\right), & \left(\partial / \partial w_{J}^{2}\right)=\left(e_{2} I\right)+i\left(e_{2} K\right), \\
\left(\partial / \partial z_{K}^{1}\right)=e_{1}+i\left(e_{1} K\right), & \left(\partial / \partial w_{K}^{1}\right)=\left(e_{1} I\right)-i\left(e_{1} J\right), \\
\left(\partial / \partial z_{K}^{2}\right)=e_{2}+i\left(e_{2} K\right), & \left(\partial / \partial w_{K}^{2}\right)=\left(e_{2} I\right)-i\left(e_{2} J\right) .
\end{array}
$$

Given a 2-vector of type $(1,1)$, with respect to a complex structure, it is the sum of complex lines. Thus each 2 -vector of the form, say, $\left(\partial / \partial z^{1}\right) \wedge\left(\partial / \partial \bar{z}^{2}\right)$, 
can be expressed as a sum of complex lines. Now

$$
\begin{aligned}
& \operatorname{Re}\left(\partial / \partial z_{J}^{1}\right) \wedge\left(\partial / \partial \bar{z}_{J}^{2}\right)+\operatorname{Re}\left(\partial / \partial z_{K}^{1}\right) \wedge\left(\partial / \partial \bar{z}_{K}^{2}\right)-\operatorname{Re}\left(\partial / \partial w_{I}^{1}\right) \wedge\left(\partial / \partial \bar{w}_{I}^{2}\right) \\
&= e_{1} \wedge e_{2}+\left(e_{1} J\right) \wedge\left(e_{2} J\right)+e_{1} \wedge e_{2} \\
&+\left(e_{1} K\right) \wedge\left(e_{2} K\right)-\left(e_{1} J\right) \wedge\left(e_{2} J\right)-\left(e_{1} K\right) \wedge\left(e_{2} K\right) \\
&= 2 e_{1} \wedge e_{2}
\end{aligned}
$$

completing the proof of (4.8).

Any 4-plane in $\mathbf{H}^{n}$ can be expressed as $\xi \wedge \eta$ with $\xi$ and $\eta 2$-planes. Using (4.8),

$$
\begin{aligned}
\xi \wedge \eta= & \left(\sum a_{j} \wedge I a_{j}+\sum b_{j} \wedge J b_{j}+\sum c_{j} \wedge K c_{j}\right) \\
& \wedge\left(\sum a_{j}^{\prime} \wedge I a_{j}^{\prime}+\sum b_{j}^{\prime} \wedge J b_{j}^{\prime}+\sum c_{j}^{\prime} \wedge K c_{j}^{\prime}\right) .
\end{aligned}
$$

Expanding this product, note that each term, say for example $a_{j} \wedge I a_{j} \wedge b_{k}^{\prime} \wedge$ $J b_{k}^{\prime}$, is a 4-plane contained in a quaternionic 2-plane $\left(\operatorname{span}_{\mathbf{H}}\left\{a_{j}, b_{k}^{\prime}\right\}\right.$ in the example).

\section{THE GENERALIZED ASSOCIATIVE CALIBRATION}

Just as the fundamental 4-form $\Phi_{K}$ on $\mathbf{H}^{n}$ introduced in the last section generalizes the Cayley form $\boldsymbol{\Phi}$ on $\mathbf{H}^{2} \cong \mathbf{O}$, the 3-form, $\phi_{K}$ on $\operatorname{Im} \mathbf{H} \oplus \mathbf{H}^{n-1}$, examined in this section, generalizes the associative form $\phi$ on $\operatorname{Im} \mathbf{H} \oplus \mathbf{H} \cong$ $\operatorname{Im} \mathbf{O}$.

The associative form $\phi \in \Lambda^{3}(\operatorname{Im} \mathbf{O})^{*}$ is defined by

$$
\phi(x, y, z) \equiv\langle x, y z\rangle \text { for all } x, y, z \in \operatorname{im} \mathbf{O} \text {. }
$$

or in coordinates,

$$
\phi \equiv \omega^{234}-\omega^{265}+\omega^{735}+\omega^{764}-\omega^{278}+\omega^{368}+\omega^{458}
$$

This 3-form $\phi$ is a calibration which attains its maximum value one on those 3-planes $\xi \equiv x \wedge y \wedge z$ in $\operatorname{Im} \mathbf{O}$ which are associative, i.e. $x(y z)=(x y) z$ (see [HL]).

Note that

$$
\phi=1\lrcorner \Phi
$$

where $1 \in \mathbf{O}$.

The coassociative form $\psi \in \Lambda^{4}(\operatorname{Im} \mathbf{O})^{*}$ is defined by

$$
\psi(x, y, z, w) \equiv \frac{1}{2}\langle x, y(\bar{z} w)-w(\bar{z} y)\rangle \quad \text { for all } x, y, z, w \in \operatorname{Im} \mathbf{O} .
$$

That is,

$$
\left.\psi \equiv \Phi\right|_{\operatorname{Im} O},
$$

is just the Cayley form $\Phi$ restricted to $\operatorname{Im} \mathbf{O}$.

Thus

$$
\Phi=1^{*} \wedge \phi+\psi
$$

(see $[\mathrm{HL}])$. 
Definition 5.4. The 3-form $\phi_{K} \in \Lambda^{3}\left(\operatorname{Im} \mathbf{H} \oplus \mathbf{H}^{n-1}\right)^{*}$ defined by

$$
\left.\phi_{K} \equiv e_{1}\right\lrcorner \Phi_{K},
$$

where $e_{1} \equiv(1,0, \ldots, 0) \in \mathbf{H}^{n}$, is the generalized associative form.

Note that

$$
\Phi_{K}=\omega^{1} \wedge \phi_{K}+\psi_{K}
$$

Remark. Since $\Phi_{K}$ is fixed by $\operatorname{Sp}(n)$ and $\operatorname{Sp}(n)$ acts transitively on the unit sphere $\left.S^{4 n-1} \subset \mathbf{H}^{n} ; e_{1}\right\lrcorner \Phi_{K}$ and $\left.u\right\lrcorner \Phi_{K}$ are $\mathrm{SO}(4 n)$ equivalent for any unit vector $u \in \mathbf{H}^{n}$.

Let $I, J, K$ denote the standard orthonormal basis for $\operatorname{Im} \mathbf{H}$ and $I^{*}, J^{*}$, $K^{*}$ the dual basis of 1 -forms on $\operatorname{Im} \mathbf{H}$.

Proposition 5.7. The generalized associative form can be expressed as

$$
\phi_{K}=I^{*} \wedge J^{*} \wedge K^{*}-I^{*} \wedge \omega_{I}-J^{*} \wedge \omega_{J}+K^{*} \wedge \omega_{K},
$$

where $\omega_{I}, \omega_{J}, \omega_{K} \in \Lambda^{2} \mathbf{H}^{n-1}$ are the Kähler forms determined by $I, J$, and $K$ respectively. Similarly, the generalized coassociative form can be expressed as

$$
\psi_{K}=J^{*} \wedge K^{*} \wedge \omega_{I}+K^{*} \wedge I^{*} \wedge \omega_{J}-I^{*} \wedge J^{*} \wedge \omega_{K}-\frac{1}{2} \omega_{I}^{2}-\frac{1}{2} \omega_{J}^{2}+\frac{1}{2} \omega_{K}^{2} .
$$

Proof. Substituting into (2.22) with $1^{*} \equiv \omega^{1}, I^{*} \equiv \omega^{2}, J^{*} \equiv \omega^{3}, K^{*} \equiv \omega^{4}$, yields

$$
\begin{aligned}
\Phi_{K}= & -\frac{1}{2}\left(1^{*} \wedge I^{*}-J^{*} \wedge K^{*}+\omega_{I}\right)^{2} \\
& -\frac{1}{2}\left(1^{*} \wedge J^{*}-K^{*} \wedge I^{*}+\omega_{J}\right)^{2} \\
& +\frac{1}{2}\left(1^{*} \wedge K^{*}-I^{*} \wedge J^{*}+\omega_{K}\right)^{2} .
\end{aligned}
$$

Expanding this expression out and comparing with the formula

$$
\Phi_{K}=1^{*} \wedge \phi_{K}+\psi_{K}
$$

proves the desired formulas for $\phi_{K}$ and $\psi_{K}$.

Definition 5.11. If $L$ is a quaternionic line in $\mathbf{H}^{n-1}$, let

$$
\operatorname{ASOC}(\operatorname{Im} \mathbf{H} \oplus L) \equiv\left\{\xi \in G_{\mathbf{R}}(3, \operatorname{Im} \mathbf{H} \oplus L): \phi_{K}(\xi)=|\xi|\right\}
$$

and

$$
\operatorname{COAS}(\operatorname{Im} \mathbf{H} \oplus L) \equiv\left\{\xi \in G_{\mathbf{R}}(4, \operatorname{Im} \mathbf{H} \oplus L): \psi_{K}(\xi)=|\xi|\right\} .
$$

Theorem 5.12. The generalized associative form $\phi_{K} \in \Lambda^{3}\left(\operatorname{Im} \mathbf{H} \oplus \mathbf{H}^{n-1}\right)^{*}$ and the generalized coassociative form $\psi_{K} \in \Lambda^{4}\left(\operatorname{Im} \mathbf{H} \oplus \mathbf{H}^{n-1}\right)^{*}$ are both calibrations. Moreover, $\phi_{K}(\xi)=1$ if and only if $\xi \in \mathrm{ASOC}(\operatorname{Im} \mathbf{H} \oplus L)$ for some quaternionic line $L \subset \mathbf{H}^{n-1}$; and $\psi_{K}(\xi)=1$ if and only if $\xi \in \operatorname{COAS}(\operatorname{Im} \mathbf{H} \oplus L)$ for some quaternionic line $L \subset \mathbf{H}^{n-1}$.

Theorem 5.12 is an immediate consequence of Theorem 2.27. 
Theorem 5.13. The associative calibration $\phi_{K} \in \Lambda^{3}\left(\operatorname{Im} \mathbf{H} \oplus \mathbf{H}^{n-1}\right)^{*}$ and the coassociative calibration $\psi_{K} \in \Lambda^{4}\left(\operatorname{Im} \mathbf{H} \oplus \mathbf{H}^{n-1}\right)^{*}$ are both exposed and hence maximal.

Proof. Suppose $\phi^{\prime} \in \Lambda^{3}\left(\operatorname{Im} \mathbf{H} \oplus \mathbf{H}^{n-1}\right)^{*}$ is another calibration and that

$\operatorname{ASOC}\left(\operatorname{Im} \mathbf{H} \oplus \mathbf{H}^{n-1}\right) \subset G\left(\phi^{\prime}\right) \equiv\left\{\xi \in G_{\mathbf{R}}\left(3, \operatorname{Im} \mathbf{H} \oplus \mathbf{H}^{n-1}\right): \phi^{\prime}(\xi)=1\right\}$.

Then we must show that $\alpha \equiv \phi_{K}-\phi^{\prime}$ vanishes. First consider the case $\phi_{K} \in$ $\Lambda^{3}\left(\operatorname{Im} \mathbf{H} \oplus \mathbf{H}^{2}\right)^{*}$ when $n=3$, and let $e_{1}, e_{2}, e_{3}$ denote the standard quaternionic basis for $\mathbf{H}^{3}$. Let $\xi$ denote one of the real axis 3-planes in $\operatorname{Im} \mathbf{H} \oplus \mathbf{H}^{2}$. If $\xi \in \mathrm{ASOC}\left(\operatorname{Im} \mathbf{H} \oplus \mathbf{H}^{2}\right)$ then $\alpha(\xi)=0$ since $\phi_{K}=\phi^{\prime}(\xi)=1$. Suppose $\xi \notin$ $\operatorname{ASOC}\left(\operatorname{Im} \mathbf{H} \oplus \mathbf{H}^{2}\right)$.

Case $1 . \xi$ is a three plane in $\{0\} \times \mathbf{H}^{2}$. Then $\operatorname{span} \xi$ must contain at least two real axes in the same quaternion axis. Thus $\operatorname{span} \xi$ must contain a complex line with respect to at least one of the complex structures $I, J$, or $K$. Say $\xi=e_{2} \wedge I e_{2} \wedge u$. Then $\eta \equiv-I e_{1} \wedge e_{2} \wedge I e_{2}$ is a first cousin of $\xi$ which is associative ( $e_{1} \wedge \eta$ is Cayley). Therefore, by the first cousin principle, both $\phi_{K}$ and $\phi^{\prime}$ must vanish on $\xi$.

Case 2. $\xi=u \wedge \eta$ with $u \in\left\{I e_{1}, J e_{1}, K e_{1}\right\}$, say $u=I e_{1}$. Again, it is easy to see that $\xi$ has an associative first cousin.

Thus each axis 3-plane $\xi$ in $\operatorname{Im} \mathbf{H} \oplus \mathbf{H}^{2}$ is either associative or has an axis first cousin that is associative. This proves that, for general $n, \alpha=\phi_{K}-\phi^{\prime}$ vanishes on all 3-planes $\xi$ in $\operatorname{Im} \mathbf{H} \oplus V \subset \operatorname{Im} \mathbf{H} \oplus \mathbf{H}^{n-1}$ where $V$ is a quaternionic plane in $\mathbf{H}^{n-1}$.

Now suppose $\xi$ is an axis 3-plane in $\operatorname{Im} \mathbf{H} \oplus \mathbf{H}^{n-1}$. If $\operatorname{span} \xi$ is not contained in $\{0\} \times \mathbf{H}^{n-1}$ then, since it is 3-dimensional, $\operatorname{span} \xi \subset \operatorname{Im} \mathbf{H} \oplus V$ for some quaternionic plane $V$. Thus we may assume $\xi$ is an axis 3-plane in $\{0\} \times \mathbf{H}^{3}$. Choose coordinates $z^{1}, w^{1}, z^{2}, w^{2}, z^{3}, w^{3}$ for $\mathbf{H}^{3} \cong \mathbf{C}^{6}$ with respect to the complex structure $I$. The difference $\alpha=\phi_{K}-\phi$ belongs to $\operatorname{Re} \Lambda^{3,0}\left(\mathbf{C}^{6}\right)$. The remainder of the proof, which is similar to the proof of Lemma 4.7, is omitted.

To prove that the coassociative form $\psi_{K} \in \Lambda^{4}\left(\operatorname{Im} \mathbf{H} \oplus \mathbf{H}^{n-1}\right)^{*}$ is maximal, suppose $\psi^{\prime} \in \Lambda^{4}\left(\operatorname{Im} \mathbf{H} \oplus \mathbf{H}^{n-1}\right)^{*}$ is another calibration which is identically 1 on COAS. Since $\psi_{K}$, restricted to $\{0\} \times \mathbf{H}^{n-1}$, is a generalized Cayley form, it is maximal on $\{0\} \times \mathbf{H}^{n-1}$. Therefore, $\alpha \equiv \psi_{K}-\psi^{\prime}$ vanishes on all 4-planes in $\{0\} \times \mathbf{H}^{n-1}$.

Also, employing the first cousin principle, $\alpha$ vanishes on $\xi$ if $\xi$ contains a complex line with respect to one of the complex structures $I, J$, or $K$. Therefore, $\alpha=I^{*} \wedge \beta_{I}+J^{*} \wedge \beta_{J}+K^{*} \wedge \beta_{K}$, with $\beta_{I}, \beta_{J}, \beta_{K} \in$ $\Lambda^{3}\left(\{0\} \times \mathbf{H}^{n-1}\right)^{*}$. These forms can be shown to be in $\Lambda^{3,0}\left(\mathbf{C}^{2 n-2}\right)$, for each of the complex structures. Then, using complex coordinates, and arguing as in the proof of Lemma 4.7, the proof that $\alpha=0$ is completed. The details are omitted. 


\section{6. $\operatorname{Sp}(n)$-INVARIANT CALIBRATIONS}

In this section we consider the 4-forms $\Phi(A) \equiv \widetilde{A} \in \Lambda^{4}\left(\mathbf{H}^{n}\right)^{*}$ in the algebra generated by $\omega_{I}, \omega_{J}$, and $\omega_{K}$ (see $(2.6)$ for the definition of $\left.\tilde{A} \equiv \Phi(A)\right)$. Recall that $A \in \operatorname{Sym}_{\mathbf{R}}(3)$ is a real symmetric $3 \times 3$ matrix. Each $\omega_{I}, \omega_{J}$, $\omega_{K}$ and hence each $\Phi(A)$ is fixed by $\operatorname{Sp}(n)$. The objective of this section is to determine for which $A$ the form $\Phi(A)$ is a calibration, i.e.,

$$
\Phi(A)(\xi) \leq 1 \text { for all } \xi \in G_{\mathbf{R}}\left(4, \mathbf{H}^{n}\right) .
$$

Consider, as usual, the unit scalars $\mathrm{Sp}(1)$ acting on $\mathbf{H}^{n}$ on the right. Recall that the induced action of $\operatorname{Sp}(1)$ on the image of $\Phi$ : $\operatorname{Sym}_{\mathbf{R}}(3) \rightarrow \Lambda^{4}\left(H^{n}\right)^{*}$ is given by

$$
R_{u}^{*}[\Phi(A)]=\Phi\left(g_{u} A g_{u}^{t}\right), \quad u \in \mathrm{Sp}(1),
$$

where $g_{u}(x) \equiv u x \bar{u}$ for all $x \in \operatorname{Im} H$. Note that $\chi: \operatorname{Sp}(1) \rightarrow \operatorname{SO}(3)$ defined by $u \mapsto g_{u} \equiv \chi(u)$ is the usual double cover of the special orthogonal group, for $\mathbf{R}^{3} \cong \operatorname{Im} \mathbf{H}$, by the unit quaternions $\operatorname{Sp}(1)$.

Each $A \in \operatorname{Sym}_{\mathbf{R}}(3)$ can be put in diagonal form. That is, for some $g_{u} \in$ $\mathrm{SO}(3), \lambda \equiv g_{u} A g_{u}^{t}$ is a diagonal $3 \times 3$ matrix, with diagonal entries $\lambda \equiv$ $\left(\lambda_{1}, \lambda_{2}, \lambda_{3}\right)$. Since $\operatorname{Sp}(1) \subset \mathrm{SO}(4 n)$, the 4 -form $\Phi(A)$ is a calibration if and only if $R_{u}^{*}[\Phi(A)]$ is a calibration. Therefore, we need only consider the special (diagonal) 4-forms:

$$
\Phi(\lambda) \equiv \lambda_{1} \frac{1}{2} \omega_{I}^{2}+\lambda_{2} \frac{1}{2} \omega_{J}^{2}+\lambda_{3} \frac{1}{2} \omega_{K}^{2} .
$$

Theorem 6.3. The 4-form $\Phi(\lambda) \in \Lambda^{*}\left(H^{n}\right)^{*}$ is a calibration if and only if $\lambda \equiv$ $\left(\lambda_{1}, \lambda_{2}, \lambda_{3}\right) \in \mathbf{R}^{3}$ lies in the convex body defined by $\left|\lambda_{j}\right| \leq 1, j=1,2,3$ and $\left|\lambda_{1}+\lambda_{2}+\lambda_{3}\right| \leq 1$. See Figure 2 .

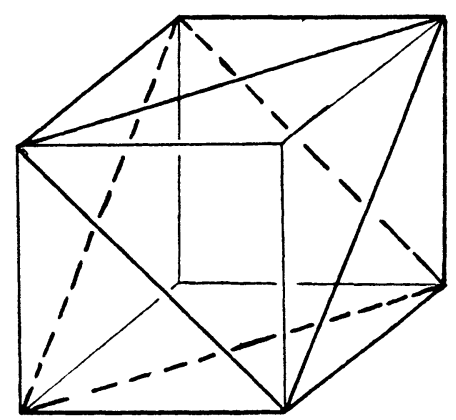

FIGURE 2

Remark. For example, $\lambda_{2} \frac{1}{2} \omega_{J}^{2}+\lambda_{3} \frac{1}{2} \omega_{K}^{2}$ is a calibration if and only if $\left(\lambda_{2}, \lambda_{3}\right)$ lies in the convex body (see Figure 3 ) defined by $\left|\lambda_{2}\right|+\left|\lambda_{3}\right| \leq 1$ if $\lambda_{2} \lambda_{3} \geq 0$, and by $\max \left\{\left|\lambda_{2}\right|,\left|\lambda_{3}\right|\right\} \leq 1$ if $\lambda_{2} \lambda_{3} \leq 0$. 


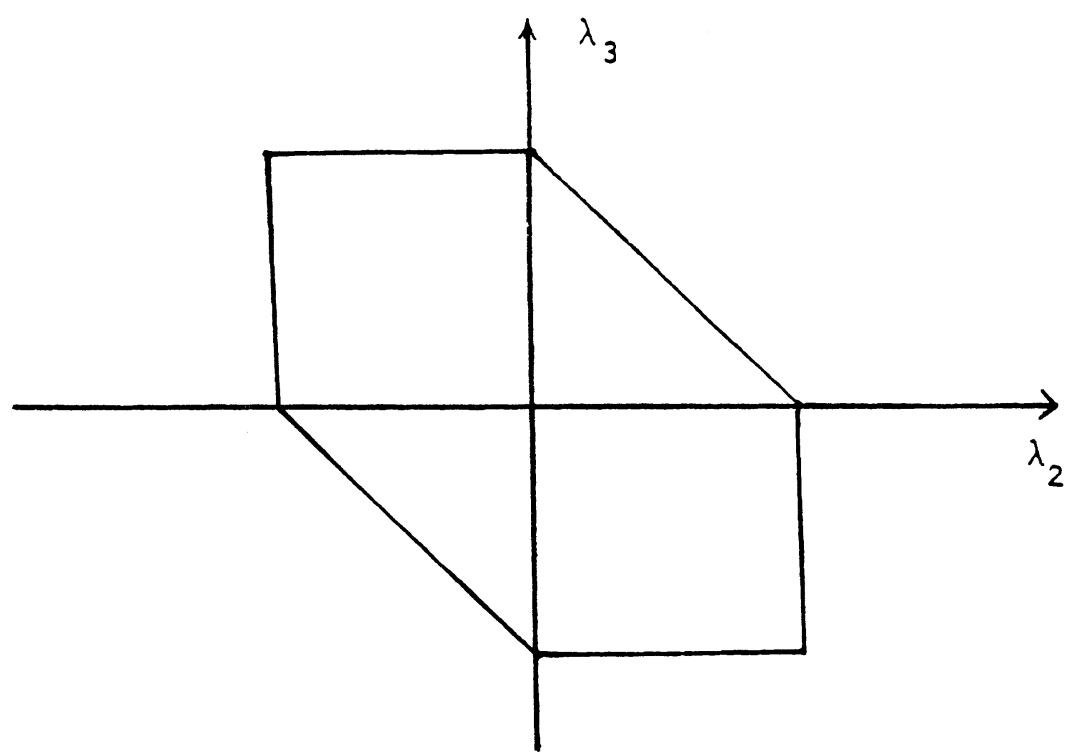

Figure 3

Two calibrations $\phi$ and $\psi$ are considered the same type if there exists an orthogonal transformation $g$ with $\psi=g^{*} \phi$. If $n=2$ see Dadok, Harvey, and Morgan [DHM] for the classification of the $\Phi(\lambda)$.

Theorem 6.4 (Classification $n \geq 3$ ). The calibrations $\Phi(\lambda)$ with $\lambda$ on the boundary of the convex body described in Theorem 6.3 can be classified as follows.

(6.5) Vertices (Cayley): $\Phi_{I}, \Phi_{J}, \Phi_{K}$ correspond to the vertices with two of the $\lambda_{1}, \lambda_{2}, \lambda_{3}$ equal to -1 and one equal +1 . The group $\operatorname{Sp}(n) \cdot\left(S^{1} \cup R_{I} S^{1}\right)$ fixes $\Phi(\lambda)$.

(6.6) Vertices (anti-Cayley): $-\Phi_{I},-\Phi_{J},-\Phi_{K}$ correspond to the vertices with two of the $\lambda_{1}, \lambda_{2}, \lambda_{3}$ equal to +1 and one equal -1 .

(6.7) Edges (Kähler): The midpoints of these edges yield the calibrations $\frac{1}{2} \omega_{I}^{2}$, $\frac{1}{2} \omega_{J}^{2}, \frac{1}{2} \omega_{K}^{2}$. The group $U(2 n)$ fixes these calibrations.

(6.8) Edges (anti-Kähler): The midpoints of these edges yield the calibrations $-\frac{1}{2} \omega_{I}^{2},-\frac{1}{2} \omega_{J}^{2},-\frac{1}{2} \omega_{K}^{2}$

(6.9) Edges (special isotropic): The midpoints of each of the six nontrivial edges joining a Cayley vertex to an anti-Cayley vertex yield the calibrations $\operatorname{Re} \frac{1}{2} \sigma^{2}$, where $\sigma$ is a complex symplectic form on $\mathbf{H}^{n} \cong \mathbf{C}^{2 n}$ corresponding to one of the six complex structures $\pm I, \pm J, \pm K$. For example, with $+I, \sigma=\omega_{J}-i \omega_{K}$ (see (2.37) and Theorem 2.38). The group $\mathrm{Sp}(n)$ fixes these calibrations. 
(6.10) Face (quaternionic): The midpoint of this face yields the quaternionic 4-form

$$
\alpha \equiv \frac{1}{3}\left(\frac{1}{2} \omega_{I}^{2}+\frac{1}{2} \omega_{J}^{2}+\frac{1}{2} \omega_{K}^{2}\right) \in \Lambda^{4}\left(\mathbf{H}^{n}\right)^{*} .
$$

This calibration distinguishes the quaternionic lines in $\mathbf{H}^{n}$. That is, $G(\alpha) \equiv$ $\left\{\xi \in G_{\mathbf{R}}\left(4, \mathbf{H}^{n}\right): \alpha(\xi)=1\right\}$ is just $P^{n-1}(\mathbf{H})$, quaternionic projective space.

The quaternionic unitary group $\mathrm{Sp}(n) \cdot \mathrm{Sp}(1)$ fixes $\alpha$.

(6.12) Face (antiquaternionic): The midpoint of this face is the calibration $-\alpha$, which distinguishes the quaternionic lines with reverse orientation.

(6.13) Faces ( $K$-complex and $\sigma$-isotropic): The midpoints of these six faces yield calibrations of the form

$$
\beta \equiv \frac{1}{2} \omega_{K}^{2}-\frac{1}{3}\left(\frac{1}{2} \omega_{I}^{2}+\frac{1}{2} \omega_{J}^{2}\right) .
$$

The contact set

$$
G(\beta) \equiv\left\{\xi \in G_{\mathbf{R}}\left(4, \mathbf{H}^{n}\right): \beta(\xi)=1\right\}
$$

consists of those real 4-planes $\xi$ which are both $K$-complex and $\sigma \equiv \omega_{I}-i \omega_{J}$ isotropic. The group $\mathrm{Sp}(n) \cdot\left(S^{1} \cup R_{I} S^{1}\right)$ fixes $\beta$.

Proof of Theorem 6.3. Each of the six vertices of the convex body defined in Theorem 6.3 has comass one. This is exactly Theorem 2.27 if the vertex is $\Phi_{K} \equiv \Phi(-1,-1,+1)$; and the other vertices are \pm 1 times a calibration of this type. Consequently, each $\Phi(\lambda)$ in the convex hull of these six vertices also has comass one.

To prove that each $\Phi(\lambda)$, with $\lambda$ outside the convex hull, has comass strictly larger than one, rescale by $0<t<1$ so that $t \Phi(\lambda)$ is on the boundary of the convex hull. Now note that (see the statement of the Classification Theorem 6.4) there exist $\xi \in G_{\mathbf{R}}\left(4, \mathbf{H}^{n}\right)$ with $t \Phi(\lambda)(\xi)=1$. Therefore $\Phi(\lambda)(\xi)=t^{-1}>1$.

The proof of the Classification Theorem, which is routine but somewhat tedious, is omitted.

\section{The generality of $\Phi_{K}$-MANIFolds}

In this section, we want to make some remarks on the "generality" of the submanifolds in $\mathbf{H}^{n}$ calibrated by $\Phi_{K}$.

We begin by listing some of the "obvious" methods of producing $\Phi_{K}$-manifolds. First, if $V \subseteq \mathbf{H}^{n}$ is any quaternionic 2-plane, then $\Phi_{K} \mid V$ is equivalent to the Cayley calibration $\Phi$ on $\mathbf{O}$ (see $\S 2$ ). According to [HL], if $\Sigma^{3} \subseteq \mathbf{O}$ is any real analytic submanifold of dimension 3 in $\mathbf{O}$, there exists a unique (real analytic) 4-manifold $M^{4} \subseteq \mathbf{O}$ which satisfies $\Sigma^{3} \subseteq M^{4}$ and is a $\Phi$ manifold. This method produces (locally) all of the $\Phi_{K}$-manifolds $M^{4} \subseteq \mathbf{H}^{n}$ which lie linearly fully in some quaternion 2-plane $V \subseteq H^{n}$. Let us call these $\Phi_{K}$ manifolds Cayley. A second method is to note that if $I_{\theta}=(\cos \theta) I+(\sin \theta) J$ is used to induce a complex structure on $\mathbf{H}^{n}$, then any $I_{\theta}$-complex 2-manifold 
$M \subseteq \mathbf{H}^{n}$ is a $\Phi_{K}$-manifold once we reverse the orientation. Of course, there are many complex surfaces in $\mathbf{C}^{2 n}$ which lie linearly fully in $\mathbf{C}^{2 n}$. Thus, this second method produces many examples which cannot be generated by the first method when $n>2$. Let us call these $\Phi_{K}$-manifolds anti- $I_{\theta}$-complex. A third method is to consider the $K$-complex surfaces $M \subseteq \mathbf{H}^{n}$ which are isotropic with respect to the $K$-holomorphic 2-form $\sigma=\omega_{I}-\sqrt{-1} \omega_{J}$. It is easy to see that these are quite general. For example, if we choose $K$-complex coordinates $z^{1}, \ldots, z^{n}, w^{1}, \ldots, w^{n}$ on $H^{n} \cong \mathbf{C}^{2 n}$ for which

$$
\sigma=d z^{1} \wedge d w^{1}+d z^{2} \wedge d w^{2}+\cdots+d z^{n} \wedge d w^{n},
$$

then a holomorphic map $\psi: \mathbf{C}^{2} \rightarrow \mathbf{C}^{2 n}$ satisfies $\psi^{*}(\sigma)=0$ iff $\psi=\left(z^{\alpha}(u, v)\right.$; $\left.w^{\alpha}(u, v)\right)$ satisfies the single first order equation

$$
\sum_{\alpha=1}^{n} \frac{\partial z^{\alpha}}{\partial u} \frac{\partial w^{\alpha}}{\partial v}-\frac{\partial z^{\alpha}}{\partial v} \frac{\partial w^{\alpha}}{\partial u}=0
$$

This equation obviously has many solutions. If a solution $\psi$ is an immersion, then $\psi\left(\mathbf{C}^{2}\right) \subseteq \mathbf{C}^{2 n}\left(\cong \mathbf{H}^{n}\right)$ is a $\Phi_{K}$-manifold. Let us refer to these $\Phi_{K}$ submanifolds as $K$-complex $\sigma$-isotropic surfaces.

Although it is not remarkable that the submanifolds of these three types, Cayley, $I_{\theta}$-anticomplex, and $K$-complex $\sigma$-isotropic can be calibrated (see $\S 6$ ), perhaps it is surprising that all these types can be calibrated simultaneously. In particular, any union of 4-manifolds without boundary in $H^{n}$ of these types is mass-minimizing. It remains to be seen whether there exist $\Phi_{K}$-manifolds in $\mathbf{H}^{n}$ which do not fall into one of these types.

In studying this question, we will need tools from the theory of moving frames and differential systems. It turns out that a special case must be disposed of separately from the general argument to be considered further on.

Proposition 7.1. Suppose that $M^{4} \subseteq \mathbf{H}^{n}$ is a connected smooth 4-manifold with the property that there exists a smooth map $\theta: M^{4} \rightarrow \mathbf{R} / 2 \pi \mathrm{Z}$ so that $T_{p} M^{4}$ is an $I_{\theta(p)}$-complex 2-plane for all $p \in M$. Then either $M^{4}$ is an (open subset of) a quaternion line in $H^{n}$ or else $\theta$ is constant.

Proof. If $M^{4}$ is a quaternion line in $\mathbf{H}^{n}$, there is nothing to prove. Thus, we may assume that, at least on a dense open subset, $T_{p} M^{4}$ is not a quaternion line in $\mathbf{H}^{n}$. We now construct a differential system appropriate for our problem. Let us expand standard quaternion coordinates as $q^{l}=x^{l}+i y^{l}+j u^{l}+k v^{l}$ $(1 \leq l \leq n)$. It is easy to see that the forms

$$
\begin{gathered}
\omega^{\prime}=d x^{l}+i\left(\cos \psi d y^{\prime}+\sin \psi d u^{\prime}\right), \\
\eta^{\prime}=d v^{l}-i\left(\sin \psi d y^{\prime}-\cos \psi d u^{\prime}\right)
\end{gathered}
$$

are of type $(1,0)$ with respect to the complex structure $I_{\psi}$ for all $\psi \in \mathbf{R} / 2 \pi \mathbf{Z}$. In fact, $\left\{\omega_{\psi}, \eta_{\psi}^{l} \mid l=1, \ldots, n\right\}$ spans $\Lambda_{\psi}^{1,0}\left(\mathbf{H}^{n}\right)\left(=\right.$ the $(1,0)$-forms on $\mathbf{H}^{n}$ using the complex structure $I_{\psi}$ ). 
Now let $X=\mathbf{H}^{n} \times(\mathbf{R} / 2 \pi \mathbf{Z})$ and let $I$ be the differential system on $X$ generated a $(p, \theta)$ by the forms in $\Lambda_{\theta}^{3,0}\left(\mathbf{H}^{n}\right)$. Note that we may "graph" $M^{4} \subseteq \mathbf{H}^{n}$ to get a new 4-manifold $\widetilde{M}^{4} \subseteq \mathbf{H}^{n} \times(\mathbf{R} / 2 \pi \mathbf{Z})=X$ by setting

$$
\widetilde{M}^{*}=\left\{(p, \theta(p)) \mid p \in M^{4}\right\} .
$$

Since any element of $\Lambda_{\theta(p)}^{3,0}\left(H^{n}\right)$ must vanish on $T_{p} \widetilde{M}^{4}$ for all $p \in \widetilde{M}^{4}$ (after all, $T_{p} M^{4}$ is an $I_{\theta(p)}$-complex 2-plane), we see that $\widetilde{M}^{4}$ is an integral manifold of $I$. Because of the presence of $\psi$ in $\omega_{\psi}$ and $\eta_{\psi}^{l}$, these forms are not closed. In fact

$$
d \omega_{\psi}=\frac{1}{2} d \psi \wedge\left(\eta_{\psi}^{l}-\bar{\eta}_{\psi}^{l}\right), \quad d \eta_{\psi}=-\frac{1}{2} d \psi \wedge\left(\omega_{\psi}^{l}-\bar{\omega}_{\psi}^{l}\right) .
$$

In particular, we have

$$
\begin{aligned}
d\left(\omega_{\psi}^{1} \wedge \omega_{\psi}^{2} \wedge \eta_{\psi}^{1}\right) & \equiv \frac{1}{2} d \psi \wedge\left(-\bar{\eta}_{\psi}^{1} \wedge \omega_{\psi}^{2} \wedge \eta_{\psi}^{1}-\omega_{\omega}^{1} \wedge \bar{\eta}_{\psi}^{2} \wedge \eta_{\psi}^{1}+\omega_{\psi}^{1} \wedge \omega_{\psi}^{2} \wedge \bar{\omega}_{\psi}^{1}\right) \\
d\left(\omega_{\psi}^{1} \wedge \eta_{\psi}^{1} \wedge \eta_{\psi}^{2}\right) & \equiv \frac{1}{2} d \psi \wedge\left(-\bar{\eta}_{\psi}^{1} \wedge \eta_{\psi}^{1} \wedge \eta_{\psi}^{2}+\omega_{\psi}^{1} \wedge \bar{\omega}_{\psi}^{1} \wedge \eta_{\psi}^{2}+\omega_{\psi}^{1} \wedge \eta_{\psi}^{1} \wedge \bar{\omega}_{\psi}^{2}\right)
\end{aligned}
$$

where the congruences are taken modulo $\Lambda_{\psi}^{3.0} \subseteq I$.

Using the normal form derived in $\S 3$ and the natural extension of the action of $H$ on $X$, we may suppose that $(p, \theta(p))=(0,0) \in X$ and that

$$
T_{p} M^{4}=u_{1} \wedge u_{2} \wedge u_{3} \wedge u_{4}=\xi
$$

where

$$
\begin{array}{ll}
u_{1}=(\cos \phi) \partial / \partial x^{1}+(\sin \phi) \partial / \partial x^{2}, & u_{2}=(\cos \phi) \partial / \partial y^{1}+(\sin \phi) \partial / \partial y^{2}, \\
u_{3}=(\cos \phi) \partial / \partial u^{1}-(\sin \phi) \partial / \partial u^{2}, & u_{4}=(\cos \phi) \partial / \partial v^{1}-(\sin \phi) \partial / \partial v^{2},
\end{array}
$$

where $0<\phi \leq \pi / 4$ (we may assume $\phi \neq 0$ since $T_{p} M$ is not a quaternion line). Since we are at a point where $\psi=0$, we may compute

$$
\begin{gathered}
0=\left.d\left(\omega_{\psi}^{1} \wedge \omega_{\psi}^{2} \wedge \eta_{\psi}^{1}\right)\right|_{\dot{\xi}}=\left(\left.d \theta\right|_{p}\right) \wedge(-2 i) \cos ^{2} \phi \sin \phi\left(u_{1}^{*}+i u_{2}^{*}\right) \wedge u_{3}^{*} \wedge u_{4}^{*}, \\
0=\left.d\left(\omega_{\psi}^{1} \wedge \eta_{\psi}^{1} \wedge \eta_{\psi}^{2}\right)\right|_{\dot{\xi}}=\left(\left.d \theta\right|_{p}\right) \wedge(2 i) \cos ^{2} \phi \sin \phi u_{1}^{*} \wedge u_{2}^{*} \wedge\left(u_{4}^{*}+i u_{3}^{*}\right) .
\end{gathered}
$$

This uses

$$
\begin{array}{ll}
\omega_{\psi \mid \tilde{\xi}}^{1}=(\cos \phi)\left(u_{1}^{*}+i u_{2}^{*}\right), & \omega_{\psi \mid \dot{\xi}}^{2}=(\sin \phi)\left(u_{1}^{*}+i u_{2}^{*}\right), \\
\eta_{\psi \mid \dot{\xi}}^{1}=(\cos \phi)\left(u_{4}^{*}+i u_{3}^{*}\right), & \eta_{\psi \mid \tilde{\xi}}^{2}=(-\sin \phi)\left(u_{4}^{*}+i u_{3}^{*}\right)
\end{array}
$$

and the natural identification $T_{p} M \cong T_{(p, \theta(p))} \widetilde{M}=\tilde{\xi}$. Since $u_{1}^{*}, u_{2}^{*}, u_{3}^{*}, u_{4}^{*}$ form a basis of $T_{p} M$, it follows that $\left.d \theta\right|_{p}=0$. Since $p$ was arbitrary, it follows that $d \theta=0$ on $M^{4}$. Thus $\theta$ is constant.

Let us now consider the general case of an oriented 4-manifold $M^{4} \subseteq \mathbf{H}^{n}$. We can define a symmetric matrix of functions $A=\left(a_{i j}\right)$ on $M^{4}$ as follows. 
For each $p \in M$, let $\xi(p) \in \Lambda^{4}\left(T_{p} M\right)$ denote the unit oriented volume element. We now define (see (2.6)):

$$
\begin{aligned}
& {\left[\begin{array}{lll}
a_{11}(p) & a_{12}(p) & a_{13}(p) \\
a_{21}(p) & a_{22}(p) & a_{23}(p) \\
a_{31}(p) & a_{32}(p) & a_{33}(p)
\end{array}\right]} \\
& =\frac{1}{2}\left[\begin{array}{ccc}
\omega_{I}^{2}(\xi(p)) & \omega_{I} \wedge \omega_{J}(\xi(p)) & \omega_{I} \wedge \omega_{K}(\xi(p)) \\
\omega_{J} \wedge \omega_{I}(\xi(p)) & \omega_{J}^{2}(\xi(p)) & \omega_{J} \wedge \omega_{K}(\xi(p)) \\
\omega_{K} \wedge \omega_{I}(\xi(p)) & \omega_{K} \wedge \omega_{J}(\xi(p)) & \omega_{K}^{2}(\xi(p))
\end{array}\right]
\end{aligned}
$$

These quantities on $M$ are invariant under the motion of $\mathbf{H}^{n}$ induced by translations and rotations by $\operatorname{Sp}(n)$. Of course, $M^{4}$ is a $\Phi_{K}$-manifold iff $a_{33}-a_{11}-a_{22}=1$. Note that the quantities

$$
b_{1}=-a_{33}, \quad b_{2}=\sqrt{\left(a_{11}-a_{22}\right)^{2}+4 a_{12}^{2}}
$$

are actually invariant under $\mathbf{H}$, the stabilizer of $\Phi_{K}$. Moreover, $b_{1}$ and $b_{2}^{2}$ are actually smooth functions on $M^{4}$. Note that if we put $\xi(p)$ in "normal form" as in Theorem 2.27

$$
\xi(p)=\left(c_{1} e_{1}+s_{1} e_{s}\right) \wedge\left(c_{2} e_{2}+s_{2} e_{6}\right) \wedge\left(c_{2} e_{3}-s_{2} e_{7}\right) \wedge\left(c_{1} e_{4}-s_{1} e_{8}\right)
$$

then we may compute

$$
\begin{aligned}
& b_{1}=\frac{1}{2}\left(\cos \left(2\left(\phi_{1}+\phi_{2}\right)\right)+\cos \left(2\left(\phi_{1}-\phi_{2}\right)\right)\right), \\
& b_{2}=\frac{1}{2}\left|\cos \left(2\left(\phi_{1}+\phi_{2}\right)\right)-\cos \left(2\left(\phi_{1}-\phi_{2}\right)\right)\right| .
\end{aligned}
$$

Since we have the inequalities $0 \leq \phi_{2} \leq \phi_{1} \leq \pi / 2$ and $\phi_{1}+\phi_{2} \leq \pi / 2$, we get

$$
\cos \left(2\left(\phi_{1}-\phi_{2}\right)\right)=b_{1}+b_{2}, \quad \cos \left(2\left(\phi_{1}+\phi_{2}\right)\right)=b_{1}-b_{2} .
$$

Since $0 \leq \phi_{1}-\phi_{2}, \phi_{1}+\phi_{2} \leq \pi / 2$, we may invert to get

$$
\begin{aligned}
& \phi_{1}=\frac{1}{4}\left(\cos ^{-1}\left(b_{1}+b_{2}\right)+\cos ^{-1}\left(b_{1}-b_{2}\right)\right), \\
& \phi_{2}=\frac{1}{4}\left(\cos ^{-1}\left(b_{1}-b_{2}\right)-\cos ^{-1}\left(b_{1}+b_{2}\right)\right) .
\end{aligned}
$$

It follows that $\phi_{1}$ and $\phi_{2}$ are continuous functions on any $\Phi_{K}$-manifold $M^{4}$. If $\phi_{1} \equiv \phi_{2}$, then we are in the situation covered by the previous proposition. Since we are going to discard this case, we will assume from now on that $\phi_{1}>$ $\phi_{2}$ on $M^{4}$. Also the case where $\phi_{1} \equiv \pi / 2$ and $\phi_{2} \equiv 0$ corresponds to the $\Phi_{K}$-manifolds which are $K$-complex $\sigma$-isotropic. Thus, we shall assume that $\phi_{1}<\pi / 2$.

Since any $\Phi_{K}$-manifold is minimal, it is necessarily real analytic. It is therefore easy to see that the remaining possibilities (i.e., not $I_{\theta}$-anticomplex and not $K$-complex $\sigma$-isotropic) have dense open sets where one of the following possibilities holds:

(1) $\phi_{2} \equiv 0,0<\phi_{1}<\pi / 2$ (bottom edge of $\phi$-triangle). 
(2) $\phi_{2} \equiv \pi / 2-\phi_{1}$ and $\pi / 4<\phi_{1}<\pi / 2$ (upper right edge of $\phi$-triangle).

(3) $0<\phi_{2}<\min \left(\phi_{1}, \pi / 2-\phi_{1}\right)$ (interior of $\phi$-triangle).

In each case, the stabilizer of $\xi_{\phi}$ in $H$ has a constant dimension. For example, in Case 3 (the generic case), the stabilizer of $\xi_{\phi}$ is a circle in $H$.

From now on, we assume that our $\Phi_{K}$-manifold $M^{4} \subseteq \mathbf{H}^{n}$ falls into one of these cases. To study such manifolds, we introduce the moving frame. Let $\underline{E}_{1}, \ldots, \underline{E}_{n}$ denote the standard quaternion basis of $\mathbf{H}^{n}$. We introduce the standard real basis of $\mathbf{H}^{n}, \underline{e}_{1}, \ldots, \underline{e}_{4 n}$ by defining $\underline{e}_{4 p-3}=\underline{E}_{p}, \underline{e}_{4 p-2}=\underline{E}_{p} i$, $\underline{e}_{4 p-1}=\underline{E}_{p} j, \underline{e}_{4 p}=\underline{E}_{p} k$ for $p=1, \ldots, n$. Let $\mathscr{F}\left(=\operatorname{Sp}(n) \cdot\left(S^{1} \cup R_{i} S^{1}\right)\right)$ denote the set of bases $e=\left(e_{1}, \ldots, e_{4 n}\right)$ which are obtainable from $\underline{e}$ by rotations in $H=\mathrm{Sp}(n) \cdot\left(S^{1} \cup R_{i} S^{1}\right)$. By the usual theory of the moving frame, we may write $d\left(e_{1}, \ldots, e_{4 n}\right)=\left(e_{1}, \ldots, e_{4 n}\right) \beta$ where $\beta$ is a matrix of 1 -forms with values in the Lie algebra of $H$. We can give an explicit description of $\beta$ as follows: It will be convenient to identify the quaternions with a $4 \times 4$ real matrix algebra as follows:

$$
x+i y+j z+k w=\left[\begin{array}{rrrr}
x & -y & -z & -w \\
y & x & -w & z \\
z & w & x & -y \\
w & -z & y & x
\end{array}\right]
$$

Let us set

$$
\hat{k}=\left[\begin{array}{rrrr}
0 & 0 & 0 & -1 \\
0 & 0 & 1 & 0 \\
0 & -1 & 0 & 0 \\
1 & 0 & 0 & 0
\end{array}\right]
$$

(Note that $\hat{k}$ commutes with $i, j$, and $k$.) Then we may write $\beta$ in the form

$$
\beta=\left[\begin{array}{ccc}
\beta_{11} & \cdots & \beta_{1 n} \\
\vdots & & \vdots \\
\beta_{n 1} & \cdots & \beta_{n n}
\end{array}\right]
$$

where each $\beta_{m l}$ is a $4 \times 4$ block satisfying

(i) $\beta_{m l}+{ }^{t} \beta_{l m}=0$.

(ii) $\beta_{m l}$ has values in $\mathbf{H}$ for $m \neq l$.

(iii) $\beta_{m m}=\beta_{0} \hat{k}+\beta_{m m}^{\prime}$ where $\beta_{m m}^{\prime}$ has values in $\mathbf{H}$.

(Note that if we set $\beta_{0}=0$ then we get the Lie algebra of $\operatorname{Sp}(n)$. )

Now let $x: M^{4} \rightarrow \mathbf{H}^{n}$ be an immersion of a $\Phi_{K}$-manifold satisfying our genericity hypothesis above. Then there exists a framing $e: M^{4} \rightarrow \mathscr{F}$ (at least locally) which satisfies for all $p \in M$

$$
\overrightarrow{T_{p} M}=u_{1}(p) \wedge u_{2}(p) \wedge u_{3}(p) \wedge u_{4}(p)
$$


where

$$
\begin{aligned}
& u_{1}(p)=\cos \phi_{1}(p) e_{1}(p)+\sin \phi_{1}(p) e_{5}(p), \\
& u_{2}(p)=\cos \phi_{2}(p) e_{2}(p)+\sin \phi_{2}(p) e_{6}(p), \\
& u_{3}(p)=\cos \phi_{2}(p) e_{3}(p)-\sin \phi_{2}(p) e_{7}(p), \\
& u_{4}(p)=\cos \phi_{1}(p) e_{4}(p)-\sin \phi_{1}(p) e_{8}(p) .
\end{aligned}
$$

If we let $\alpha^{1}, \alpha^{2}, \alpha^{3}, \alpha^{4}$ denote the coframe of $M^{4}$ dual to the tangent frame field $u_{1}, u_{2}, u_{3}, u_{4}$, then setting $\omega^{m}=e_{m} \cdot d x$, we get

$$
\begin{gathered}
\omega^{1}=\cos \phi_{1} \alpha^{1}, \quad \omega^{2}=\cos \phi_{2} \alpha^{2}, \quad \omega^{3}=\cos \phi_{2} \alpha^{3}, \quad \omega^{4}=\cos \phi_{1} \alpha^{4}, \\
\omega^{5}=\sin \phi_{1} \alpha^{1}, \quad \omega^{6}=\sin \phi_{2} \alpha^{2}, \quad \omega^{7}=-\sin \phi_{2} \alpha^{3}, \quad \omega^{8}=-\sin \phi_{1} \alpha^{4}, \\
\omega^{m}=0 \quad \text { for } m>8 .
\end{gathered}
$$

A tedious calculation using $d \omega^{m}=0$ shows that, for $p>2$, there exist quaternion functions $u_{p}, v_{p}$ on $M$ so that

$$
\begin{gathered}
\beta_{p 1}=u_{p} \sin \phi_{1}\left(\alpha^{1}+k \alpha^{4}\right)+v_{p} \sin \phi_{2}\left(\alpha^{2}+k \alpha^{3}\right), \\
\beta_{p 2}=\left[-v_{p} \cos \phi_{1}\left(\alpha^{1}+k \alpha^{4}\right)+u_{p} \cos \phi_{2}\left(\alpha^{2}+k \alpha^{3}\right)\right] i
\end{gathered}
$$

(This strongly uses the assumptions $\phi_{1}>\phi_{2}$ and $\phi_{1}<\pi / 2$. In the cases $\phi_{1}=$ $\phi_{2}\left(I_{\theta}\right.$-anticomplex) and $\phi_{1}=\pi / 2$ ( $K$-complex $\sigma$-isotropic), these identities definitely fail.) Note that $u_{p} \equiv v_{p} \equiv 0$ is equivalent to the condition that $e_{1} \wedge e_{2} \wedge e_{3} \wedge e_{4} \wedge e_{5} \wedge e_{6} \wedge e_{7} \wedge e_{8}$ be constant on $M$, i.e., that $M$ lie in a quaternion 2-plane in $\mathbf{H}^{n}$ and hence be of Cayley type.

This suggests the following formulation of our problem as a differential system. Let $X=\mathscr{F} \times \mathbf{H}^{n} \times T^{2}$. Let $x: X \rightarrow \mathbf{H}^{n}$ be projection on the second factor and let $\left(\phi_{1}, \phi_{2}\right)$ denote the (angular) coordinates on $T^{2}$. We regard $\beta$ as being well defined on $X$. In fact, by pull-back, we may regard the $e_{m}: \mathscr{F} \rightarrow \mathbf{H}^{n}$ as $\mathbf{H}^{n}$-valued functions on $X$. We define $\omega^{m}=\left\langle e_{m}, d x\right\rangle$ as usual. Define 1 -forms on $X$

$$
\begin{aligned}
& \left(\begin{array}{l}
\alpha^{1} \\
\alpha^{2} \\
\alpha^{3} \\
\alpha^{4}
\end{array}\right)=\left(\begin{array}{c}
\cos \phi_{1} \omega^{1}+\sin \phi_{1} \omega^{5} \\
\cos \phi_{2} \omega^{2}+\sin \phi_{2} \omega^{6} \\
\cos \phi_{2} \omega^{3}-\sin \phi_{2} \omega^{7} \\
\cos \phi_{1} \omega^{4}-\sin \phi_{1} \omega^{8}
\end{array}\right), \\
& \left(\begin{array}{l}
\theta^{1} \\
\theta^{2} \\
\theta^{3} \\
\theta^{4}
\end{array}\right)=\left(\begin{array}{c}
-\sin \phi_{1} \omega^{1}+\cos \phi_{1} \omega^{5} \\
-\sin \phi_{2} \omega^{2}+\cos \phi_{2} \omega^{6} \\
\sin \phi_{2} \omega^{3}+\cos \phi_{2} \omega^{7} \\
\sin \phi_{1} \omega^{4}+\cos \phi_{1} \omega^{8}
\end{array}\right) .
\end{aligned}
$$

Let $\mathbf{H}^{2(n-2)}$ have quaternion valued coordinates $\left\{u_{p}, v_{p} \mid p=3, \ldots, n\right\}$. On $Y=X \times \mathbf{H}^{2(n-2)}$ define the forms

$$
\begin{gathered}
\theta_{p 1}=\beta_{p 1}-\left(u_{p} \sin \phi_{1}\left(\alpha^{1}+k \alpha^{4}\right)+v_{p} \sin \phi_{2}\left(\alpha^{2}+k \alpha^{3}\right)\right) \\
\theta_{p 2}=\beta_{p 2}-\left(-v_{p} \cos \phi_{1}\left(\alpha^{1}+k \alpha^{4}\right)+u_{p} \cos \phi_{2}\left(\alpha^{2}+k \alpha^{3}\right)\right) i
\end{gathered}
$$


Now on $Y$ consider the differential system $I$ generated by $\left\{\theta^{1}, \theta^{2}, \theta^{3}, \theta^{4}\right.$, $\left.\omega^{9}, \omega^{10}, \ldots, \omega^{4 n}, \theta_{p 1}, \theta_{p 2}\right\}$ (where $p=3, \ldots, n$ ). Let the independence conditions be given by $\alpha=\alpha^{1} \wedge \alpha^{2} \wedge \alpha^{3} \wedge \alpha^{4} \neq 0$. Then we have shown that the integrals of $(I, \alpha)$ on $Y$ correspond to framed $\Phi_{k}$-manifolds which are not either $I_{\theta}$-anticomplex or $K$-complex $\sigma$-isotropic.

It is easy to see that, when restricted to $X \times\{0\} \subseteq Y$, this system becomes involutive with characters $s_{1}^{\prime}=s_{2}^{\prime}=s_{3}^{\prime}=4, s_{4}^{\prime}=0$. This gives rise to the $\Phi_{k}$-manifolds of Cayley type.

Unfortunately, the system $(I, \alpha)$ on $Y$ is not involutive. In fact, in general, the torsion is not absorbable. Involved calculations suggest that, except for very special possibilities, this system has no integrals except those which lie on $X \times\{0\}$, i.e., Cayley type solutions. We have not been able to prove this, however, as the algebraic difficulties involved in analyzing the sysiem become too formidable. The upshot of the calculations is that, if $\Phi_{k}$-manifolds exist which are not of one of the three types, Cayley, $I_{\theta}$-anticomplex, or $K$-complex $\sigma$-isotropic, then they are quite special. In particular, they depend on at most two functions of three variables. Further progress in understanding this problem will be reported on as it arises.

\section{HYPER-KÄHLER MANIFOLDS}

The appropriate general setting for the fundamental calibration $\Phi_{K}$ is obtained by replacing $\mathbf{H}^{n}$ by a hyper-Kähler manifold $X$.

Suppose $V$ is a right quaternion vector space, i.e., the scalars, $\mathbf{H}$, act on $V$ on the right. A function $\varepsilon: V \times V \rightarrow \mathbf{H}$ is said to be quaternionic hermitian if $\varepsilon$ is real bilinear and

$$
\varepsilon(x, y \lambda)=\varepsilon(x, y) \lambda, \quad \varepsilon(x \lambda, y)=\bar{\lambda} \varepsilon(x, y),
$$

for all $x, y \in V$ and all scalars $\lambda \in H$. If, in addition,

$$
\overline{\varepsilon(x, y)}=\varepsilon(y, x) \text { for all } x, y \in V
$$

then $\varepsilon$ is said to be quaternion hermitian symmetric.

The quaternionic hermitian form $\varepsilon$ is said to be nondegenerate if $\varepsilon(x, y)=0$ for all $y \in V$ implies $x=0$ and $\varepsilon(x, y)=0$ for all $x \in V$ implies $y=0$. Moreover, $\varepsilon$ is said to be positive if $\varepsilon(x, x)>0$ for all nonzero $x \in V$.

Now assume that $V$ is equipped with a nondegenerate quaternionic hermitian symmetric form $\varepsilon$. (The standard model is $V \equiv \mathbf{H}^{n}$ and $\varepsilon(x, y) \equiv$ $\sum_{l=1}^{n} \bar{x}_{l} y_{l}$.) Exactly as in $\S 2$, each unit imaginary quaternion $u \in S^{2} \subset \operatorname{Im} \mathbf{H}$ determines a complex structure $R_{u}$ on $V$ (by right multiplication) and a Kähler form $\omega_{u}(x \wedge y) \equiv \operatorname{Re} \varepsilon(x u, y) \equiv\langle u, \varepsilon(x, y)\rangle$. Here $\langle$,$\rangle denotes the standard$ real inner product on $\mathbf{H}$.

There are several (equivalent) ways of defining a hyper-Kähler manifold. The next definition contains, in a certain sense, the maximal amount of information. 
Definition 8.3. An n-dimensional hyper-Kähler manifold $X, \varepsilon$ is a smooth $4 n$ dimensional real manifold $X$ equipped with the following extra structure. First, assume that the quaternions $\mathbf{H}$ act (smoothly) on the tangent bundle on the right giving each tangent space, $T_{p} X$, the structure of a right quaternionic vector space. Second, suppose $\varepsilon$ is a positive quaternionic hermitian symmetric bilinear form on each tangent space $T_{p} X$ which varies smoothly with the point $p \in X$. Thus $\operatorname{Re} \varepsilon$ provides $X$ with a Riemannian structure. Third, assume that each of the almost complex structures $R_{u}$, with $u \in S^{2} \subset \operatorname{Im} \mathbf{H}$, is integrable and that the associated 2-form $\omega_{u}$ is parallel with respect to the Levi-Civita connection associated to $\operatorname{Re} \varepsilon$.

Remark 8.4. Given the first two assumptions, namely

(1) the right action of $H$ on $T X$, and

(2) the positive $H$-hermitian symmetric bilinear form $\varepsilon$,

the third assumption can be weakened considerably. In fact, if each of the 2-forms $\omega_{u}$, with $u \in S^{2} \subset \operatorname{Im} \mathbf{H}$, is closed then automatically each of the almost complex structures $R_{u}$, is integrable,

and

each 2-form $\omega_{u}$ is parallel.

If an almost complex manifold is equipped with a closed, never vanishing $(n, 0)$-form then it is easy to verify that the Nijnhuis tensor of the almost complex structure vanishes. Hence, by the Newlander-Nirenberg theorem, the almost complex structure of such a manifold is integrable. Statement (8.5) follows from this principle applied to the $(n, 0)$-form $\sigma^{n}$ where $\sigma$ is defined by (8.7) below.

Identify the quaternion scalars $\mathbf{H} \equiv \mathbf{C} \oplus j \mathbf{C}$ with two copies of the complex numbers. Then a quaternionic hermitian symmetric form $\varepsilon$ on $V$ can be decomposed into a pair of complex valued bilinear forms $h, \sigma$

$$
\varepsilon \equiv h+j \sigma
$$

with $h$ a complex hermitian symmetric form and $\sigma$ a pure complex skew form. Here

$$
h=\langle,\rangle+i \omega_{I} \text { and } \sigma=\omega_{J}-i \omega_{K},
$$

exactly as for the standard model $\mathbf{H}^{n}$. The details are omitted.

Thus, on a hyper-Kähler manifold $X$, if the complex structure $I$ is fixed, this determines

$$
\text { a Kähler form } \omega_{I} \text {, and a closed holomorphic }(2,0) \text {-form } \sigma
$$
which is nondegenerate.

Moreover, $h$ and $\sigma$ are compatible in that

$$
h\left(R_{j} x, y\right)=\sigma(x, y) \text { for all } x, y .
$$


and

$$
\sigma\left(R_{j} x, R_{j} y\right)=\overline{\sigma(x, y)} \text { for all } x, y .
$$

Conversely, if $h$ and $\sigma$ are given and (8.10) is used to define an $I$-complex antilinear map $R_{j}$ then one can show that $\sigma\left(R_{j} x, R_{j} y\right)=\overline{\sigma(x, y)}$, for all $x, y$ if and only if $R_{j}^{2}=-1$. Thus,

(8.12). If $X$ is a $2 n$-dimensional Kähler manifold equipped with a closed holomorphic $(2,0)$-form $\sigma$ which is nondegenerate and compatible with the given hermitian metric $h$ then $\varepsilon \equiv h+j \sigma$ defines a hyper-Kähler structure on $X$.

Even if $h$ and $\sigma$ are not compatible, $X$ may admit a hyper-Kähler metric.

Proposition 8.13. Suppose $X$ is a compact manifold. If $X$ is a Kähler manifold equipped with a holomorphic symplectic form $\sigma$ then $X$ admits a hyper-Kähler structure $\varepsilon$, of the form $\varepsilon=h+j \sigma$ for some hermitian form $h$ on $X$.

Proof. Since $\sigma^{n}$ is a never vanishing holomorphic volume form, the first Chern class, $c_{1}(x)$, must vanish. Consequently the Calabi-Yau Theorem states that $X$ admits a homologous Kähler metric $h$ which is Ricci flat. Moreover, $\sigma$ must be parallel with respect to this metric, because of the Bochner identity.

The proof is completed as follows. Note that in this next result $X$ is not assumed to be compact or simply connected.

Proposition 8.14. Suppose $X$ is both a Kähler manifold (with hermitian form $h$ ) and a holomorphic symplectic manifold (with symplectic form $\sigma$ ), and that $\sigma$ is parallel.

Then $X$ admits a hyper-Kähler metric $\varepsilon$ which can be chosen to be either of the form $\varepsilon=\tilde{h}=j \sigma$ or $\varepsilon=h+j \tilde{\sigma}$ for some $\tilde{h}$ and $\tilde{\sigma}$.

To prove this proposition, first note that $\sigma$ can be put in canonical form with respect to $h$ (at each point)

$$
\sigma \equiv \lambda_{1} Z_{1} \wedge Z_{2}+\cdots+\lambda_{n} Z_{2 n-1} \wedge Z_{2 n}
$$

with $\lambda_{1} \geq \lambda_{2} \geq \cdots>\lambda_{n}>0$ and $Z_{1}, \ldots, Z_{2 n}$ a unitary basis of 1,0 vectors.

Relabel with $\mu_{1} \equiv \lambda_{1}, \ldots, \mu_{r}=\lambda_{n}$ so that $\mu_{1}>\cdots>\mu_{r}$. Then one can show that $\mu_{1}, \ldots, \mu_{r}$ are global constants on $X$ and that the corresponding eigenspaces $V_{1}, \ldots, V_{r}$ are parallel. Now either rescale $h$ to obtain $\tilde{h}$ or rescale $\sigma$ to obtain $\tilde{\sigma}$.

Of course, since $\varepsilon$ is parallel on a hyper-Kähler manifold, the holonomy group must preserve $\varepsilon$ and hence be contained in $\operatorname{Sp}(n)$. Conversely, if the holonomy group of a $4 n$-dimensional Riemannian manifold $X$ is contained in $\operatorname{Sp}(n)$ then $X$ admits a hyper-Kähler structure.

Compact examples with local holonomy actually equal to $\operatorname{Sp}(n) \quad(n \geq 2)$ were thought to be nonexistent for several years (Bogomolov [Bo]), however 
since then compact examples have been found (cf. Beauville [Be]). More recently, the method of symplectic reduction provides a rich collection of examples of hyper-Kähler manifolds, see [HKLR].

Todorov [T] has recently announced that if a simply connected compact complex manifold $X$ admits a unique (up to a constant) closed holomorphic 2-form $\sigma$ and if this 2-form $\sigma$ is nondegenerate (as a complex skew bilinear form) then $X$ admits a hyper-Kähler structure $\varepsilon$. (Once the existence of a Kähler metric on $X$ is established, it follows that $X$ admits a Calabi-Yau metric, with $\sigma$ parallel, providing the hyper-Kähler structure.)

All of the concepts and results of $\S 2$ carry over to a general hyper-Kähler manifold $X, \varepsilon$. The 4 -form $\Phi_{K}$ on $X$ is defined by

$$
\Phi_{K} \equiv-\frac{1}{2} \omega_{I}^{2}-\frac{1}{2} \omega_{J}^{2}+\frac{1}{2} \omega_{K}^{2}
$$

or equivalently by

$$
\Phi_{K} \equiv-\frac{1}{2} \omega_{I}^{2}-\operatorname{Re} \frac{1}{2} \sigma^{2}, \quad \text { with } \sigma \equiv \omega_{J}-i \omega_{K}
$$

Remark. In fact we have a 2-sphere of 4-forms $\Phi_{u}$, with $u \in S^{2} \subset \operatorname{Im} \mathbf{H}$ a unit imaginary quaternion.

The main result, Theorem 2.27 , that $\Phi_{K}$ is a calibration, carries over immediately with $\mathbf{H}^{n}$ replaced by the hyper-Kähler manifold $X$.

Definition 8.18. A real oriented 4-dimensional submanifold $M$ of a hyperKähler manifold $X$ is said to be a $\Phi_{K}$-submanifold if

$$
\Phi_{K}(\vec{M}) \equiv 1 \quad \text { at all points of } M
$$

where $\vec{M}(p)$ denotes the unit simple 4-vector $e_{1} \wedge e_{2} \wedge e_{3} \wedge e_{4}$ where $e_{1}, \ldots, e_{4}$ is an oriented orthonormal frame for $M$.

Of course, since $\Phi_{K}$ is a calibration, each $\Phi_{K}$-submanifold $M$ of a hyperKähler manifold $X$ is homologically volume minimizing.

Theorem 8.20. Suppose $X$ is a hyper-Kähler manifold. The 4-form $\Phi_{K}$ is a calibration on $X$. The $\Phi_{K}$-submanifolds of $X$ include, in particular

(1) Cayley submanifolds of a hyper-Kähler surface $Y(n=2)$ contained in $X$.

(2) $K$ complex and $\sigma \equiv \omega_{J}-i \omega_{K}$ isotropic submanifolds, i.e., complex submanifolds with respect to the complex structure $K$ on $X$, which are also $\sigma$-isotropic.

(3) $I_{\theta} \equiv \cos \theta I+\sin \theta J$ anticomplex submanifolds.

(4) Special $\theta$-isotropic submanifolds, i.e., $-\operatorname{Re} \frac{1}{2} \sigma_{\theta}^{2} \equiv-\frac{1}{2}\left(\sin \theta \omega_{I}-\cos \theta \omega_{J}\right)^{2}$ $+\frac{1}{2} \omega_{K}^{2}$-submanifolds,

here $\sigma_{\theta} \equiv-\sin \theta \omega_{I}+\cos \theta \omega_{J}-i \omega_{K}$ is a closed holomorphic 2-form with respect to the complex structure $I_{\theta}$. 


\section{REFERENCES}

[Be] A. Beauville, Variétés Kählériennes compactes avec $c_{1}=0$, Astérisque 126 (1985), 181-192.

[Bo] F. Bogomolov, Hamiltonian Kähler manifolds, Soviet Math. Dokl. 19 (1978), 1462-1465.

[BH] R. Bryant and R. Harvey, Stabilizers of calibrations (to appear).

[DHM] J. Dadok, R. Harvey, and F. Morgan, Calibrations on $\mathbf{R}^{8}$, Trans. Amer. Math. Soc. 307 (1988), 1-40.

[HL] R. Harvey and H. B. Lawson, Jr., Calibrated geometries, Acta Math. 148 (1982), 47-157.

[HKLR] N. Hitchin, A. Karlhede, U. Lindström, and M. Roček, Hyperkähler metrics and super symmetry, Comm. Math. Phys. (to appear).

[T] A. Todorov, Every holomorphic symplectic manifold admits a Kähler metric, preprint.

Department of Mathematics, Duke University, Durham, NC 27706

Department of Mathematics, Rice University, Houston, TX 77251 